\title{
Parallel response selection in dual-task situations
}

\author{
SCOTT WATTER \\ McMaster University, Hamilton, Ontario, Canada \\ and \\ GORDON D. LOGAN \\ Vanderbilt University, Nashville, Tennessee
}

\begin{abstract}
Semantic priming and response priming were studied in a dual-task procedure. In two experiments, reaction times to the first and second stimuli were faster when the finger required for the Task 1 response was the same as the finger required for the Task 2 response. Such priming suggests that Task 2 response information was generated prior to the completion of Task 1 response selection. These data pose a potential challenge to the response-selection bottleneck (RSB) theory of dual-task performance, since they may indicate a violation of the discrete-stage processing assumption on which the underlying locus-of-slack logic depends. Accommodating these data while preserving the essential bottleneck character of RSB theory may be possible but may also alter the very nature of the bottleneck itself.
\end{abstract}

Why is it so difficult to do two things at once? Many accounts of dual-task performance have suggested that the answer lies in the concept of a central processing bottleneck - a stage of processing that can only be performed for one task at a time. Using variations of the psychological refractory period (PRP) paradigm, most authors have converged on Pashler and colleagues' (Pashler, 1984, 1994; Pashler \& Johnston, 1998) response-selection bottleneck (RSB) theory. RSB theory relies on a discretestage processing assumption in modeling PRP reaction time data using the locus-of-slack logic. This assumes that the processes involved in the bottleneck - canonically the response-selection stages for both tasks in a PRP experiment-are discrete and serial. Specifically, RSB theory states that Task 2 response selection (RS) cannot begin until Task $1 \mathrm{RS}$ is complete.

This article investigates whether response-selection processes of both tasks of a dual-task pair may in fact operate in parallel, by directly testing this discreteness assumption. Instead of the more traditional locus-of-slack approach, we looked for evidence of priming of Task 1 RS processes from Task 2 RS information in a typical PRP paradigm. Such priming would imply that Task 2 RS had begun before Task 1 RS was complete - that both RS processes were operating in parallel. Such evidence would constitute a violation of the discrete-stage processing assumption and pose a problem for RSB theory. The present

This research was supported by Individual National Research Service Award F31 MH13005 from the National Institute of Mental Health to S.W. and by Grant SBR 9808971 from the National Science Foundation to G.D.L. The authors are grateful to Hal Pashler, Pierre Jolicœur, Art Kramer, and Gary Dell for helpful comments regarding this work, and to Julie Delheimer for help with data collection. Correspondence concerning this article should be addressed to S. Watter, Department of Psychology, McMaster University, 1280 Main Street West, Hamilton, ON, L8S 4K1 Canada (e-mail: watter@mcmaster.ca). study goes beyond previous investigations (e.g., Hommel, 1998; Logan \& Delheimer, 2001; Logan \& Gordon, 2001; Logan \& Schulkind, 2000) by directly assessing the influence of Task 2 response information on Task 1 response selection, independently and separately from the semantic relationship between Tasks 1 and 2 .

\section{The Psychological Refractory Period Paradigm}

Telford (1931) described the "psychological refractory period" effect in human subjects required to make responses to two stimuli in quick succession. Subjects' reaction times to the second stimulus became increasingly longer as the stimuli were presented closer together in time. Telford's analogy to the refractory behavior of action potential generation in neurons was less than ideal, but the terminology has persisted. In a typical PRP experiment, subjects are presented with two stimuli, S1 and S2, separated in time by a stimulus onset asynchrony (SOA) of $0-1,000 \mathrm{msec}$. Subjects make response R1 to S1 to produce reaction time RT1 (altogether termed Task 1) and make R2 to S2 to produce RT2 (altogether termed Task 2), typically performing the tasks in this order. The basic results from this paradigm are extremely robust. RT2 becomes longer as SOA decreases, typically approaching a slope of -1 as SOA approaches zero, but RT1 is relatively unaffected by SOA.

\section{Locus-of-Slack Logic and Response-Selection Bottleneck Theory}

In 1984, Harold Pashler described a series of experiments using the PRP paradigm that used a serial processing stage model of task performance to identify potential "bottlenecks" in dual-task PRP performance. Pashler used a generalization of Sternberg's (1969) additive-factors logic, called the locus-of-slack logic (Pashler, 1994; Pashler \& Johnston, 1998; Schweickert, 1978; Schweickert \& Townsend, 1989; Townsend \& Schweickert, 1989), to 
interpret patterns of RT1 and RT2 slowing due to manipulation of discrete stages of the tasks. The locus-of-slack logic assumes (as does its parent additive-factors logic) that individual tasks may be divided into serial stages that are discrete in the sense that a subsequent stage cannot begin until a previous stage has been completed. Stages from the two tasks in a PRP paradigm may run in parallel unless there is a bottleneck, which is defined as a processing stage that may only be performed for one task at a time. Pashler determined that such a bottleneck existed in response selection and that this accounted extremely well for the PRP effect. Subsequent research by many authors has converged on this conclusion using these methods (for reviews, see Pashler, 1994; Pashler \& Johnston, 1998). Some recent evidence suggests that this bottleneck should be extended earlier to encompass memory retrieval as well (Carrier \& Pashler, 1995; but see Logan \& Delheimer, 2001; Logan \& Schulkind, 2000). The firm logical grounding of RSB theory in the locus-of-slack logic and its robustness in explaining dual-task data make it a very strong theory indeed.

The discrete-stage processing assumption plays an important role in the mathematics that underlie the locus-ofslack logic. This assumption - that the potential bottleneck stages of both tasks in a PRP paradigm are serial and discrete with respect to each other-is the very basis from which patterns of RT additivity, under factorial manipulation of task parameters, are interpreted to determine the location of the bottleneck stage. If this discreteness assumption were dropped, it is not at all clear that the mathematics would lead to the same predictions. Indeed, if the discrete-stage processing assumption were shown to be invalid, it could pose a serious question as to the validity of RSB theory and the conclusions drawn from it.

For some readers, the notion of cascaded stage output within individual PRP tasks has potentially superseded the discrete-stage framework from which RSB theory was originally derived. It is important to note that our focus here deals only with the relationship of the two tasks' RS processes themselves. The fact remains that the interpretation of RT effects taken as evidence for RSB theory relies on the assumption that the bottleneck stages of the two tasks remain serial and discrete. Although the locus-ofslack logic most strictly assumes all stages within a single task to be serial and discrete, relaxing these assumptions to allow cascaded processing in stages other than the critical bottleneck stage, while maintaining the assumption of discrete and serial processing of the bottleneck stage between tasks, does not alter our investigation or our critical hypotheses here.

We sought to test this discreteness assumption within a standard PRP paradigm. Starting with this basic design, we assessed the potential for information generated from RS processes of each task to influence RS processes in the other. Critically, we tested whether Task 1 RS could be primed from Task 2 RS information. Such a finding would suggest that Task 2 RS had begun before Task 1 RS was complete - that is, that RS processes of both tasks were operating in parallel. If Task 2 RS begins before Task 1
RS finishes, then Task 1 and Task 2 RS cannot be discrete stages. Such a violation of the discrete-stage processing assumption would challenge the conclusions of RSB theory, given its foundation in the locus-of-slack logic.

\section{Information Crosstalk Approaches to Dual-Task Processing}

Before we consider our design and its predictions in full, we will discuss alternative approaches several other authors have taken to assessing dual-task performance, which have been instructive in arriving at our present experiments. Navon and Miller (1987) made the first strong case for a crosstalk account of dual-task interference. They demonstrated that semantic and stimulus-response (S-R) relationships within and between tasks could give rise to, and account for, a substantial amount of the performance costs associated with dual-task versus single-task performance. Hirst and Kalmar (1987) also studied such effects, manipulating the semantic relatedness of concurrently performed tasks using an auditory paradigm. More recently, several authors have taken somewhat similar approaches in order to investigate the degree of parallel task processing in dual-task performance.

Logan and colleagues used a PRP paradigm with a variety of stimuli and tasks, from letter/digit discrimination to more complex semantic judgments on whole words, to investigate crosstalk effects in dual-task processing (Logan \& Delheimer, 2001; Logan \& Gordon, 2001; Logan \& Schulkind, 2000). Logan and Schulkind's Experiment 1 required subjects to perform letter/digit discrimination on each of two stimuli in a typical PRP situation. Subjects responded to S1 and S2 with different hands and performed the tasks in strict serial order. RT1 was faster when the category of S1 (letter or digit) was the same as the category of S2 - that is, when S1 and S2 were both letters or both digits - than when one was a digit and the other was a letter. This crosstalk effect suggested that the category of S2 was retrieved while subjects were processing S1. Logan and Schulkind found the same crosstalk effects in other experiments involving either magnitude and parity judgments on digits or lexical decisions on words.

Logan and Gordon (2001) found similar crosstalk effects using a PRP paradigm. Task 2-to-Task 1 priming was observed with magnitude judgments on digits, the Stroop task, picture versus word (form) judgments, and animacy judgments on regular nouns. Logan and Delheimer (2001) also found crosstalk effects in three similar PRP experiments with episodic memory judgments. In all of these cases, RT1 was faster when S1 shared a compatible semantic or episodic relationship with S2, suggesting a substantially parallel nature for Task 1 and Task 2 central processing.

An important boundary condition for this crosstalk effect was demonstrated in Logan and Schulkind's (2000) Experiment 2. In this experiment, two single-digit stimuli were presented (the digits $1-9$, excluding 5), one above the other and separated in time by an SOA of 0-900 msec. Subjects performed one of two potential tasks on each stimulus: (1) judging whether a digit was odd or even 
(parity task) and (2) judging whether a digit was greater or less than 5 (magnitude task). In some sessions, the two tasks were the same (they used the same task set; e.g., parity-parity for Task 1-Task 2), and in others they were different (they used different task sets; e.g., magnitudeparity for Task 1-Task 2). The crosstalk effect of Task 2to-Task 1 priming was observed when Tasks 1 and 2 both used the same task set. However, when Tasks 1 and 2 used different task sets, the crosstalk effects were abolished. Logan and Gordon's (2001) Experiment 3 demonstrated the same pattern of results with form and animacy judgments on pictures and words. Strong crosstalk effects were observed with RT1 when the same judgment task was performed for both Tasks 1 and 2 (same task set for both tasks), but the crosstalk effect was all but abolished when different task sets were used for Tasks 1 and 2.

In addition to the crosstalk experiments above of Logan and colleagues, Hommel (1998) presented five dual-task experiments in which performance on a primary manual task was affected by compatibility between the secondary and primary responses and between the secondary response and the primary stimulus. Hommel suggested that a bottleneck may exist in the eventual selection of a response, but that stimulus-response translation may occur in parallel. In Hommel's Experiment 1 (for example), the letter $H$ or $S$ was presented colored red or green. Subjects had to respond to the color manually by touching a metal plate with their right or left hand as Task 1, and to respond to the letter's identity vocally by saying "right" or "left" as Task 2. Subjects were faster to respond to Task 1 when the response for Task 1 (e.g., left hand) was compatible with the response for Task 2 (e.g., saying "left").

Hommel (1998), Logan and Schulkind (2000), Logan and Delheimer (2001), and Logan and Gordon (2001) all repeatedly demonstrated crosstalk effects from Task 2 to Task 1, which might suggest that the discrete-stage processing assumption is false. However, the integrity of RSB theory can be maintained if one considers semantic and episodic memory retrieval processes to be separate from, and to occur prior to, RS processes. In this view, parallel memory retrieval for Task 1 and Task 2 may occur (as these data suggest), but response selection remains discrete and serial (but see Carrier \& Pashler, 1995).

Hommel's (1998) Experiment 1 appeared to at least partially address response-related processes, but the vital question is, at what level or stage of processing did the observed crosstalk occur? Lu and Proctor (1995) have shown evidence that S-R compatibility effects for physical stimuli (e.g., left spatial position) versus abstract stimuli (e.g., left-pointing arrow) and their compatible responses (e.g., left buttonpress) are mediated via similar (if not the same) mechanisms, indicating that stimulus information is represented via more abstract categories or cognitive codes. Similarly, abstract representation of response information is suggested by findings from Stoet and Hommel (1999), who showed that the planning of a left-hand response versus a left-foot response is interfered with equally by the planning of a separate left-hand response. Hommel, Müsseler, Aschersleben, and Prinz (2001) have suggested that response information is likely represented as cognitive response codes produced as a result of mapping stimulus codes or semantic category information via S-R translation processes.

Taken together, the studies above suggest that in Hommel's (1998) Experiment 1, the semantic representation of the stimulus category for Task 2 at least partially activated a semantic representation of the Task 2 response (i.e., a cognitive response code) prior to the completion of this same process for Task 1. Task 2-to-Task 1 response compatibility effects, presumably from interactions of the response codes for Task 1 (e.g., using the left hand) and Task 2 (e.g., saying "left"), are taken to be evidence in support of this claim. Though we do not dispute these data, we do suggest that the likely processing requirements and intermediate informational stages of Task 2 processing in this situation may afford a substantial degree of Task 2to-Task 1 influence on Task 1 response activation that is separate from, and in addition to, activation of the appropriate Task 2 response code. Specifically, in requiring verbal "left"/"right" responses to letter identity, Task 2 of Hommel's Experiment 1 likely involved the activation of more abstract conceptual representations of left and right, as well as lemmas for these response words. In this situation, these more abstract, conceptual representations of left and right information, generated as an intermediate product of Task 2 performance, may themselves be responsible for the observed influence of Task 2 processing on Task 1 response performance. The availability of multiple sources of information in Task 2 that may influence Task 1 response selection creates a potential problem for interpretation here- either semantic response code information or more abstract conceptual representations of left and right could be responsible for the priming effects seen in Task 1. In order to argue for parallel operation of response-selection processes using these cross-task priming methods, potential alternate sources of response compatibility effects must be better controlled.

Finally, Lien and Proctor (2000) also demonstrated some evidence of Task 2-to-Task 1 response compatibility in a series of three experiments primarily investigating the Simon effect (Craft \& Simon, 1970) within a PRP paradigm. This study shows perhaps the most convincing demonstration of such response-specific effects of any of the articles mentioned in this section. However, the manipulation of spatial correspondence factors throughout their experiments, plus the manipulation of compatible versus incompatible spatial mapping between rather than within subjects, adds additional potential complications for observing pure response-related interactions.

In all of the studies above, Task 2-to-Task 1 compatibility effects are either complicated by, or can be accounted for via, various stimulus category relationships between the two tasks. In order to make claims about the potential parallel operation of RS processes, we suggest that one needs to demonstrate that the RS processes of Task 1 are being influenced by information that can only be generated via Task 2 RS processes. As such, we sought to assess the potential for information generated from Task 2 
RS to influence Task 1 RS-that is, for R2 to influence R1-independent of any semantic compatibility effects of S2 on R1. Showing that a significant amount of information indicating the correct manual response to S2 has been derived before the response to $\mathrm{S} 1$ is selected would be strong evidence against discrete RS processes.

\section{The Present Study}

In order to test the discreteness assumption, we needed to look beyond the traditional locus-of-slack approach. Using methods adapted from Logan and Schulkind's (2000) Experiment 2, we developed a procedure to more critically assess crosstalk effects within a typical PRP paradigm. Our investigation differs from Hommel (1998), Logan and Schulkind (2000), Logan and Delheimer (2001), and Logan and Gordon (2001) by deliberately and explicitly designing our experiments to dissociate the cross-task priming effects of semantic stimulus and category information from the potential effects of cross-task priming of response-specific information. It is important to note that this response-specific information is likely semantic in nature, akin to the semantic response codes of Hommel et al. (2001). The distinction between the semantic representation of stimulus category and semantic representation of the correct manual response for that category is vital here, with the latter being derived from the former by task-specific rules. Whether between-task crosstalk of response information occurs at this semantic response code level, and/or whether it occurs at a later motoric level of representation, is an important consideration when attempting to distinguish the parallel operation of response-selection processes of two tasks from the parallel operation of later response-execution processes of the two tasks (among other possibilities). For our primary goals here, we consider the distinction between the task-relevant semantic category of a given stimulus (here termed semantic or semantic category information) and the semantic representation of the related manual response (here termed response information), in order to observe potential between-task crosstalk effects of this response information, independent of between-task crosstalk effects of semantic category information.

For both of our experiments, we employed magnitude and parity judgment tasks for single-digit stimuli. Subjects performed sessions in which either the same task or different tasks were used for Tasks 1 and 2. Subjects responded by pressing one of two keys for each task. The strongest and most critical test of Task 2-to-Task 1 priming of response-selection processes involved performing different tasks for Task 1 and Task 2. Using the two different task sets we employed - magnitude and parity judgments - allowed completely orthogonal stimulus-semantic-category and stimulus-response relationships. This in turn allowed the assessment of response crosstalk effects separately and independently from semantic category crosstalk effects, as is further discussed below. To better specify our assumptions and predictions, we explore the potential for cross-task priming/compatibility effects under these different-task-set conditions.
Figure 1 demonstrates an abstracted informationprocessing architecture involved with the potential crosstask semantic and response priming effects we sought to assess. Panel A demonstrates the general architecture with different tasks performed for Task 1 and Task 2, and panels $\mathrm{B}$ and $\mathrm{C}$ demonstrate the specific effects of semantic and response information within a single, example trial for Tasks 1 and 2, respectively. In all panels, both stimuli are present in the display together, representing a trial with a short SOA.

Figure 1A displays basic processing stages involved with Tasks 1 and 2. Within each single task, after encoding, stimuli are classified via semantic retrieval with regard to the rules of the current task set. This classification is in turn mapped to an appropriate motor response, which is then executed. We suggest that if the timing of Task 1 and Task 2 processes is such that information from one process is available while the like process in the other task is operating, then this information will have the potential to influence that like process in the other task. Within this framework, we identify the two potential cross-task priming effects we assessed experimentally, semantic compatibility and response compatibility.

In our experiments, when different tasks were used for Task 1 and Task 2 (i.e., magnitude judgment for one task and parity judgment for the other), the same stimulus set (digits 1 to 9, excluding 5) was mapped to completely orthogonal semantic representations for each task (odd/ even and high/low). These separate orthogonal semantic representations then mapped convergently onto the same two-choice motor responses for each task (index vs. middle finger). This allowed us to independently assess effects of semantic compatibility/priming and response compatibility/priming from one task to the other. A concrete example of these potential informational effects in a single trial is presented in panels $\mathrm{B}$ and $\mathrm{C}$ of Figure 1 for Tasks 1 and 2, respectively. Here $\mathrm{S} 1=$ "8," requiring a parity judgment, and $\mathrm{S} 2=$ " 4 ," requiring a magnitude judgment. Assuming a short SOA, both stimuli appear on screen in quick succession, with the subject selectively attending first to S1 (Figure 1B).

Let us first consider the mechanism for response-level crosstalk, in this case, where motor responses for the two tasks are the same. Considering Figure 1B, S1 (" 8 ") is classified as even via semantic retrieval, and this information is mapped to a motor response for Task 1 (index finger). Potential priming of Task 1 RS from Task 2 RS information depends on the degree to which Task 2 processing can generate its own response information (R2) in parallel with Task 1 RS. Specifically, S2 ("4") must be processed under its own task set to generate a semantic classification (low), which then determines the Task 2 motor response (also index finger). If adequate R2 information is generated before Task 1 RS is complete, in this example we may observe facilitation of the Task 1 motor response (R1) from compatible R2 information. It is essential to note that with different tasks for Task 1 and Task 2, stimuli map orthogonally to the motor responses for each task. In order to generate the systematic response 

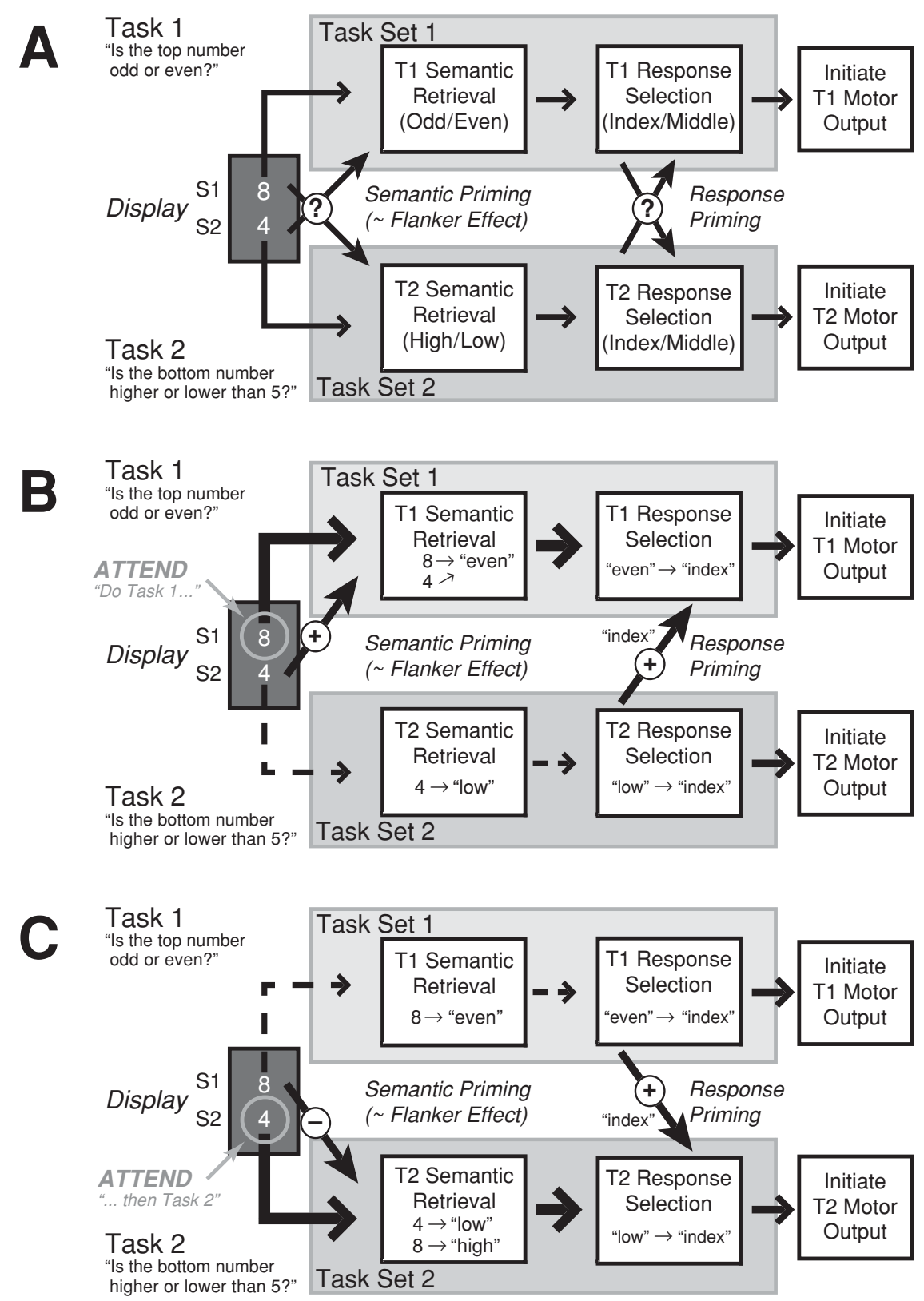

Figure 1. Mechanisms for cross-task informational priming based on Stimuli S1 and S2. Panel A shows the general architecture for the processing of both tasks, specifically here when Task 1 (T1) and Task 2 (T2) employ different task sets. Response priming (i.e., response compatibility effects) occurs when RS information from one task influences the RS process of the other. Semantic priming of stimulus classification (i.e., semantic compatibility effects) occurs when information from the other stimulus is processed along with the pertinent stimulus for a task under the current task's task set-essentially a flanker effect. Panels B and C show processing biases and information flow for a single trial with both stimuli present (and a short SOA) for Tasks 1 and 2, respectively. Potential semantic and response compatibility/priming effects for this example are described in the text. This particular trial gives an example of different semantic compatibility effects for Task 1 (compatible) and Task 2 (conflicting) arising from the same stimuli. By definition, response compatibility effects are always the same for Tasks 1 and 2 in a given trial. 
compatibility effects we assess in these studies, both stimuli must be transformed under their separate task sets. If we observe R2 influencing R1, it would suggest that sufficient R2 information must have been generated before R1 selection was complete, challenging the notion of discrete RS processes.

How can we be sure that these potential RS effects are not the result of semantic priming? There are two kinds of potential semantic priming to consider here. The first is the type that was found by Logan and Schulkind (2000), Logan and Delheimer (2001), and Logan and Gordon (2001). This kind of semantic priming involves using the same task set for Tasks 1 and 2, so that both S1 and S2 are processed by the same task set. The facilitation of RT1 via semantic compatibility effects that was observed by Logan and colleagues can be ruled out by employing different task sets for Task 1 and Task 2. The differenttask-set conditions, depicted in Figure 1, do not embody these relationships. However, a second form of semantic priming is possible in both same-task-set and differenttask-set circumstances. This involves the processing of S2 under the Task 1 task set, a process essentially equivalent to a flanker effect (Eriksen \& Eriksen, 1974). This type of priming is demonstrated in Figure $1 \mathrm{~B}$, where classification of $\mathrm{S} 1$ as even (semantic retrieval) is facilitated by consonant semantic information from $\mathrm{S} 2$ processed under the Task 1 task set.

Because both kinds of semantic priming may occur in same-task-set conditions, but only the flanker-like semantic compatibility effect may occur in different-task-set conditions, one would expect smaller semantic priming effects when different task sets are used for Task 1 and Task 2. Indeed, Logan and Schulkind (2000) and Logan and Gordon (2001) found no evidence of semantic priming in their experiments when different task sets were employed for Tasks 1 and 2, in contrast to the robust semantic compatibility effects found by these authors, as well as by Logan and Delheimer (2001), when Tasks 1 and 2 used the same task set. This suggests that the flanker-like semantic compatibility effect, should it exist, is relatively weak.

However weak this flanker-like semantic compatibility effect may be, we must ensure that we can observe and assess potential response compatibility effects separately and independently from any such semantic effects. The different-task-set conditions of our experiments were designed to accomplish precisely this discrimination. As is described above, the different tasks employed (magnitude and parity judgments) mapped the same stimulus set to orthogonal semantic representations for each task (odd/ even vs. high/low), which in turn mapped convergently to the same two-choice motor responses for each task. From this design, several relationships may be noted. S1-S2 compatibility is orthogonal to R2-R1 compatibility, and S2-R1 compatibility is also orthogonal to R2-R1 compatibility. Because of this, response compatibility effects cannot logically be derived from the semantic relationships of S2 and S1, nor can they be derived from the relationship between $\mathrm{S} 2$ and $\mathrm{R} 1$. This design allowed us to independently assess the effects of response crosstalk and flanker-like semantic crosstalk within a single PRP task using different tasks for Tasks 1 and 2. It should be noted that when the same task set is employed for both tasks, it is impossible to have such orthogonal S-R mapping. As such, same-task-set conditions do not allow independent assessment of semantic and response effects within a single subject.

The discussion above concerns the potential effects of Task 2 processing on Task 1 performance. Once subjects respond to Task 1, they are then required to perform Task 2 (Figure 1C). The same semantic compatibility process occurs again, with classification of S2 ("4") as low. Here semantic retrieval is interfered with by competing semantic information from S1 ("8") processed under the current (Task 2) task set (again, essentially a flanker effect). The response priming situation is somewhat different (and more predictable) for Task 2, since Task 1 RS has already been completed, with expected response facilitation in this case. This particular example demonstrates the potential for any combination of positive and negative semantic compatibility effects to be present for the two tasks on any given different-task-set trial. On the other hand, the nature of response compatibility means that this effect is always the same for Task 1 and Task 2 in a given trial.

Two experiments were performed to investigate the potential for parallel response selection in a typical PRP paradigm. We assessed the priming of Task 1 responseselection processes from Task 2 response information, independent of between-task semantic compatibility effects, in a design that stressed strict, serial Task 1-then-Task 2 performance. In Experiment 1, we examined performance with both the same task set and different task sets for S1 and $\mathrm{S} 2$. To better assess potential response priming effects, types of response mapping were manipulated, with some subjects responding to Task 1 and Task 2 with different hands (univalent responses) and others responding to both tasks with the same hand (bivalent responses). For both double- and single-handed conditions, response priming effects were considered on the basis of using the index versus the middle finger for response, regardless of hand (e.g., responding with the right index finger for Task 1 and the left index finger for Task 2 was considered consonant relative to the response variable). In Experiment 2, we extended the most critical quarter of Experiment 1's design, assessing different-task performance with bivalent responses, using the right versus the left hand as the response alternatives for both Task 1 and Task 2 .

Response compatibility effects of Task 1 information on Task 2 are to be expected, since Task 2 follows Task 1 in time, and are predicted to occur at all SOAs if they occur at all in a given setting. If Task 2 processing can progress sufficiently to generate $\mathrm{R} 2$ information prior to the completion of Task 1 response selection, Task 2 RS information may be able to prime Task $1 \mathrm{RS}$ processing. If such Task 2-to-Task 1 RS priming can occur, it should do so only at short SOAs and be absent at long SOAs, given the timing of stimulus presentation and the temporal overlap of critical processes. Finding such distinct effects would suggest that Task 2 RS had generated substantial 
R2 information prior to the completion of Task 1 RS. Such parallel operation of RS processes would be a violation of the discrete-stage processing assumption and could pose a substantial challenge to RSB theory.

\section{EXPERIMENT 1}

\section{Method}

\section{Subjects}

Thirty-two undergraduates from the University of Illinois at Urbana-Champaign participated in four separate $1-\mathrm{h}$ sessions. The subjects performed only one session on any day and completed the four-session experiment within 6 consecutive days. The subjects received either partial course credit or $\$ 5 / \mathrm{h}$ for their participation. All subjects were right-handed and had normal or corrected-to-normal vision.

\section{Apparatus and Stimuli}

The stimuli were the digits $1,2,3,4,6,7,8$, and 9 , presented in standard white IBM $80 \times 25$ text-screen characters on a black background. The stimuli were presented on Gateway 2000 CrystalScan 1024NI monitors, controlled by Gateway 2000486 computers. Responses to the stimuli were collected using the "." and "." keys and/or the " $z$ " and " $x$ " keys of the computer keyboard, dependent on experimental condition (described below).

Two stimuli were presented in each trial of the experiment, one above the other on the computer screen. The top stimulus (S1) always appeared first, at position [row 13, column 38] on the $80 \times$ 25 character text screen. The bottom stimulus (S2) always appeared simultaneously with or later than the top stimulus, at position [row 14, column 38]. Subjects were seated, with a screen viewing distance of approximately $60 \mathrm{~cm}$. Under these conditions, individual digit stimuli subtended $0.5^{\circ}$ vertical and $0.2^{\circ}-0.3^{\circ}$ horizontal visual angle to the subjects, depending on the stimulus, with $0.75^{\circ}$ vertical separation measured from the center of each stimulus $\left(0.25^{\circ}\right.$ vertical separation between nearest edges). The stimuli were separated in time by an SOA of $0,100,300$, or $900 \mathrm{msec}$.

The subjects were required to perform one of two predesignated tasks on each stimulus within a single trial. For a parity task, they were required to judge whether the value of the stimulus digit was odd or even. For a magnitude task, they were required to judge whether the value of the stimulus digit was greater or less than 5 (note that the digit 5 was not used as a stimulus). For any 1 -h experimental session, the tasks to be performed for S1 and S2 were fixed.

A typical trial proceeded as follows. A fixation screen was displayed for $500 \mathrm{msec}$, indicating the positions at which the two stimuli would be displayed. This display consisted of two rows of two horizontal dashes separated by three spaces, centered on the screen, indicating the central position in which each stimulus would appear. This display began at row 13 , column 36 on the screen. In the 0 -msec-SOA trials, the fixation display was replaced with a display of S1 and S2 for $1,000 \mathrm{msec}$, followed by a blank screen for $2,500 \mathrm{msec}$. In trials with SOAs greater than $0 \mathrm{msec}$, the fixation display was replaced by a display with only S1. S2 appeared on the screen accompanying S1 after the SOA duration. The two-digit display remained on the screen for $1,000 \mathrm{msec}$, followed by a blank screen for $2,500 \mathrm{msec}$.

\section{Procedure}

The experimental design involved 512 trials per 1-h session, formed from two replications of the basic 256-trial iteration ( 8 digits for $\mathrm{S} 1 \times 8$ digits for $\mathrm{S} 2 \times 4 \mathrm{SOAs}$ ), in randomized order and presented in blocks of 64 trials interleaved with rest periods. The subjects performed four of these sessions, with a different combination of tasks in each (i.e., parity-parity, magnitude-magnitude, parity-magnitude, and magnitude-parity for Task 1 and Task 2, respectively). The order of these sessions was determined according to a balanced Latin square. Prior to each 512-trial experimental session, the subjects performed an initial practice block of 64 trials, which was not considered for analysis.

The subjects indicated their responses to the stimuli by pressing the "." or the "" key and the "x" or the "z" key of the computer keyboard with the index and middle fingers of their right and left hands, respectively. Half of the subjects always made responses to both tasks with the same hand, and the other half always used one hand to respond to Task 1 and the other to respond to Task 2. Response mapping was counterbalanced between subjects for single-/doublehanded responding with the right and/or left hand, and for mapping of the high/low and odd/even responses with respect to each other and to the index or middle finger. Response mapping within Tasks 1 and 2 was kept constant for each subject across the four sessions, to minimize confusion and potential carryover effects (i.e., assignment of odd/even and high/low responses to the index or the middle finger for a specific task was held constant-mappings could conflict between Task 1 and Task 2).

The subjects were informed of the relevant task and response requirements at the beginning of each experimental session. A card displaying the current response mapping for each task was attached below the computer monitor throughout the session. The subjects were instructed to respond as quickly and as accurately as they could. Task 1 pertinence and serial performance of the tasks were emphasized at length. The subjects were told to concentrate on S1 for each trial and to make their response as quickly and as accurately as they could. Only once they had responded to S1 should they then think about S2 and try to respond quickly and accurately to it. The subjects were explicitly told not to wait until they knew both answers or to make a joint response, but to think of each trial as two completely separate tasks to be done in strict order.

\section{Data Analysis}

Mean reaction times were computed for trials in which both $\mathrm{R} 1$ and R2 were correct. Trials with reaction times longer than 3,500 msec were excluded from analysis. Data were analyzed in terms of the potential effects of separate semantic and response priming of each task by the other. For every trial, the Task 1 stimulus and task set were assessed with regard to (1) whether the Task 2 stimulus was semantically compatible or incompatible with the classification of S1 when both stimuli were considered under the Task 1 task set (akin to a flanker effect-here termed semantic compatibility); and (2) whether the motor response to Task 1 (R1) was the same as or opposite to the correct motor response to Task 2 (R2), assuming regular processing of S2 via the Task 2 task set to derive R2 (here termed response compatibility). Task 2 conditions were assessed with regard to Task 1 information for each trial in exactly the same manner. The semantic and response relations of both tasks were assessed over all SOAs for both two-handed (univalent) and one-handed (bivalent) response mappings.

Accuracy measures here represent the combined performance of both tasks, since an error in either task disqualified an entire trial from RT analyses. The accuracy measures reported for this experiment were coded via the Task 1 semantic and response conditions for each trial. Coding errors via Task 2 conditions reallocates half of the trials in each semantic category (compatible vs. incompatible) to the opposite category, but it does not reallocate trials on response classification. (Response classifications must be the same for the two tasks in each trial, since either the same or different physical motor responses were made for Tasks 1 and 2. Semantic compatibility can be different for the two tasks, depending on how S1 and S2 are classified under the two different task sets.) Accuracies were coded and assessed both ways, and little difference was observed between them, so accuracy data are only presented here coded via the Task 1 conditions, for brevity.

Separate 2 (semantic compatibility) $\times 2$ (response compatibility) $\times 4(\mathrm{SOA}) \times 2$ (single- or double-handed response) repeated measures ANOVAs were conducted for Task 1 and Task 2 reaction 
time data, for both same-task-set and different-task-set conditions. For same-task-set conditions, semantic compatibility and SOA were within-subjects factors, and response compatibility and single-/ double-handed response mapping were between-subjects factors. For different-task-set conditions, semantic compatibility, response compatibility, and SOA were within-subjects factors, and single-/ double-handed response was a between-subjects factor. The design of the same-task-set trials did not allow within-subjects counterbalancing of response compatibility with semantic compatibility. In the same-task-set trials, when the mapping of semantic responses to fingers was the same for Tasks 1 and 2 (e.g., odd = index finger and even $=$ middle finger for both tasks), responses were either semantic and response compatible or semantic and response incompatible. When response mappings were different from Task 1 to Task 2 (e.g., high $=$ index finger, low $=$ middle finger for Task 1 ; low $=$ index finger, high $=$ middle finger for Task 2 ), the two possible responses were either semantic compatible and response incompatible or response compatible and semantic incompatible. As such, response compatibility was counterbalanced between subjects for same-taskset trials, although semantic and response compatibility factors were completely counterbalanced within subjects in different-task-set trials. The combined Task 1-Task 2 accuracy data were analyzed via two separate four-way repeated measures ANOVAs identical to those for mean reaction time described above, for same-task-set and different-task-set conditions, respectively.

\section{Results}

The results for mean reaction times and accuracy are presented below, first for the same-task-set sessions (magnitude-magnitude and parity-parity for Task 1Task 2) and then for the different-task-set sessions (magnitude-parity and parity-magnitude for Task 1Task 2). Results for different response mapping types (studied between subjects) - double-handed (univalent) and single-handed (bivalent) responses for the two tasksare reported within the same- and different-task-set sections below. Overall, typical PRP effects were observed in all conditions. RT2 was observed to increase with decreasing SOA, and it approached a slope of -1 at the 0 -msec SOA. RT1 remained relatively flat with changing SOAs. Mean accuracy across all conditions was $89 \%$.

\section{Same-Task-Set Sessions}

Reaction time. Mean reaction times for same-task-set sessions for Tasks 1 and 2 are presented graphically in Figure 2. The characteristic PRP effects were observed in the same-task-set data. RT2 increased with decreasing SOA, approaching a slope of -1 at the 0 -msec SOA, whereas RT1 remained relatively flat across SOAs.

For Task 1, a distinct pattern of semantic and response priming effects was observed at the $0-\mathrm{msec}$ and $100-\mathrm{msec}$ SOAs in both single-handed (bivalent) and double-handed (univalent) conditions. Trials with both semantic and response facilitation $(\mathrm{Sem}+\mathrm{Resp}+)$ were fastest, followed by trials with both semantic and response interference $(\mathrm{Sem}-\mathrm{Resp}-)$ and with semantic facilitation/response interference (Sem+ Resp-), and semantic interference/ response facilitation trials (Sem- Resp + ) were slowest. These effects disappeared at the 300-msec and 900-msec SOAs for single-handed responses. Double-handed responses separated into double-facilitation/interference trials (Sem + Resp + and Sem- Resp -$)$, which mimicked the results for single-handed responses, and mixed-facilitation/interference trials (Sem + Resp - and Sem-Resp +), which were significantly slower than the other conditions.

Inferential statistics supported these observations for Task 1. Significant main effects were observed for semantic compatibility $[F(1,28)=23.65, p<.001]$ and SOA $[F(3,84)=10.67, p<.001]$, whose interaction was also significant $[F(3,84)=31.74, p<.001]$. A threeway interaction between response compatibility, SOA, and single-/double-handed response was also reliable $[F(3,84)=4.67, p<.01]$. Fisher's least significant difference of the mean, with an alpha of $.05\left(\mathrm{LSD}_{.05}\right)$ computed from mean square error for the highest order interaction, was $14.57 \mathrm{msec}$. By this criterion, semantic and response priming effects were significant at the 0 -msec and 100 msec SOAs for both single- and double-handed responses. Double-facilitation/interference responses were significantly different from mixed-facilitation/interference responses at the 300-msec and 900-msec SOAs for doublehanded response conditions.

These results replicate those of Logan and Schulkind (2000), Logan and Gordon (2001), and Logan and Delheimer (2001) and show an effect of Task 2 semantic information on Task 1 performance at short SOAs, which was taken as evidence of parallel memory retrieval by the authors above. R2 information also appeared to have an effect on R1 at shorter SOAs. This response-level crosstalk challenges the discrete-stage processing assumption. However, since semantic and response relationships are not completely orthogonal within subjects in the sametask-set task design, the response crosstalk effect seen here may be biased by semantic factors. The different-task-set trials provided a more rigorous test, with complete withinsubjects orthogonality of potential semantic and response crosstalk effects.

For Task 2 in the same-task-set conditions, a similar pattern of semantic and response priming effects was observed at all SOAs, in both the single- and double-handed conditions. Trials with both semantic and response facilitation $(\mathrm{Sem}+\mathrm{Resp}+)$ were fastest, followed by trials with both semantic and response interference (Sem- Resp-), with mixed-facilitation/interference trials slowest. Of this last group, semantic facilitation/response interference trials (Sem+ Resp-) were almost uniformly faster than semantic interference/response facilitation trials (SemResp+). Two-handed response conditions were uniformly slower than the corresponding one-handed conditions.

These Task 2 effects were supported by inferential statistics. Main effects were significant for SOA $[F(3,84)=$ $590.62, p<.001]$, semantic compatibility $[F(1,28)=$ $98.12, p<.001]$, and response compatibility $[F(1,28)=$ $10.40, p<.01]$. Two-way interactions between semantic and response compatibility $[F(1,28)=15.91, p<.001]$ and semantic compatibility and SOA $[F(3,84)=48.76$, $p<.001]$, as well as the three-way interaction between these variables $[F(3,84)=4.32, p<.01]$, confirmed the semantic and response priming effects and the mixedfacilitation/interference conflict cost. The observed effect of responses being generally slower in the double-handed 

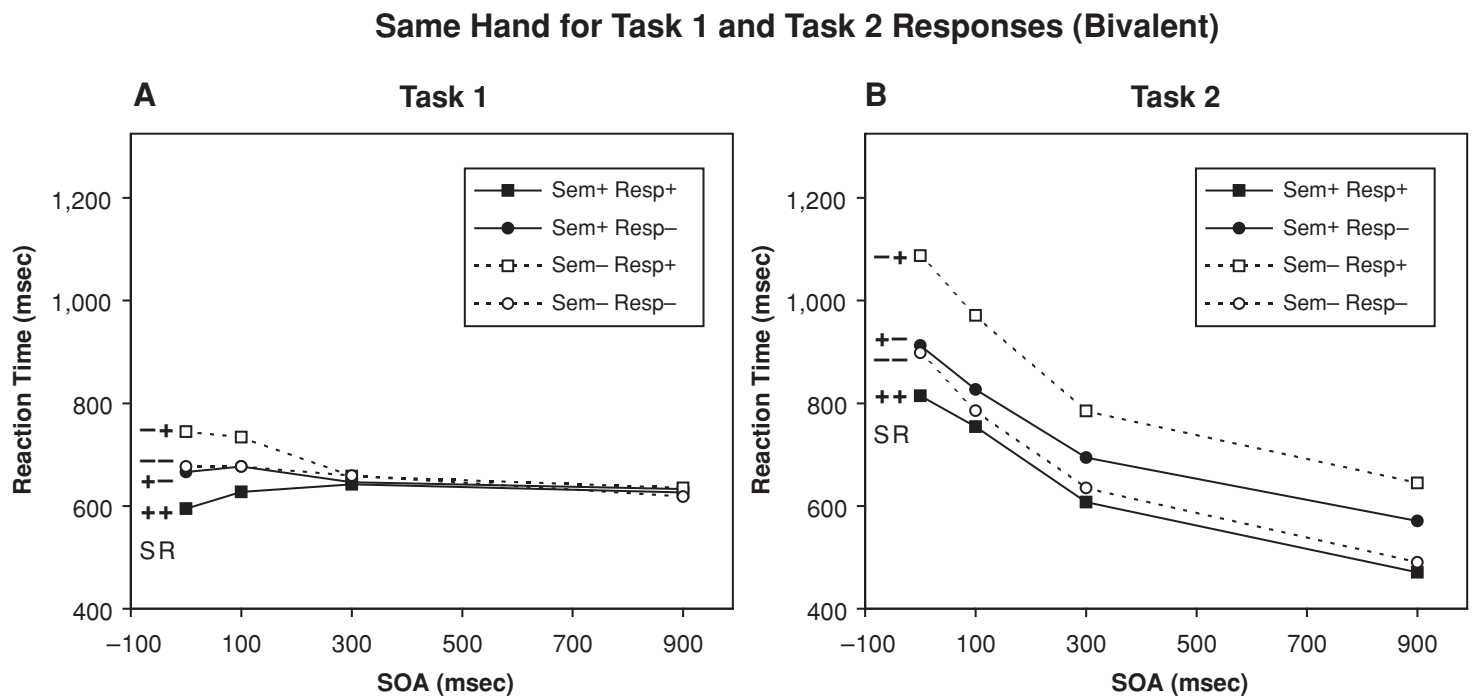

Different Hands for Task 1 and Task 2 Responses (Univalent)
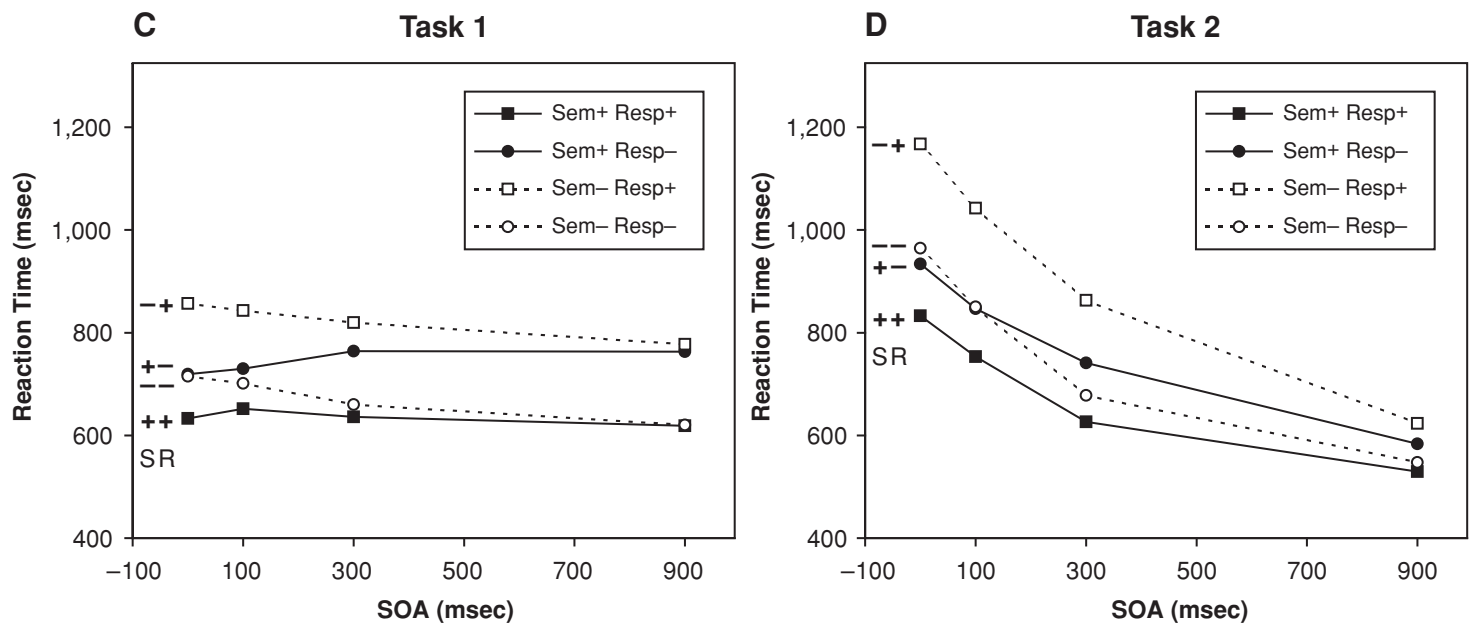

Figure 2. Experiment 1: Same-task-set mean reaction times for Tasks 1 and 2.

than in the single-handed response conditions was reflected in an additional three-way interaction between semantic compatibility, SOA, and single-/double-handed response mapping $[F(3,84)=5.59, p<.01]$. Fisher's $\operatorname{LSD}_{.05}$ for the highest order interaction was $14.71 \mathrm{msec}$. By this criterion, the semantic and response priming effects described were significant at all SOAs for Task 2 , in both the single- and double-handed response conditions.

The Task 2 findings for same-task-set trials again replicate those of Logan and Schulkind (2000), Logan and Gordon (2001), and Logan and Delheimer (2001). They demonstrate effects of semantic and response compatibility of Task 1 information on Task 2 performance. These Task 2 findings are not diagnostic with regard to the main theoretical issue, the validity of the discrete-stage processing assumption. However, they may provide additional reassurance with regard to critical Task 1 crosstalk findings, in that the pattern of significant semantic and response ef- fects on Task 2 was extremely consistent with the pattern of significant effects observed at short SOAs in Task 1.

Accuracy. Mean combined accuracy measures for the same-task-set sessions in Tasks 1 and 2 are shown in Figure 3 for single-handed (bivalent) and double-handed (univalent) responses. Accuracy performance was consistent with that for the reaction time measures. In general, higher mean error rates were observed in conditions with slower mean reaction times, with no speed-accuracy trade-off evident. Both semantic and response compatibility appeared to influence performance. In the double-handed response condition, semantic compatibility appeared to have the largest effect on overall accuracy across SOAs, with the single-handed condition showing a combination of semantic and response effects more similar to those seen in the reaction time measures.

These observations were supported by inferential statistics. Significant main effects were observed for seman- 


\section{Overall Accuracy Given Correct Response on Both Tasks}

\section{A}

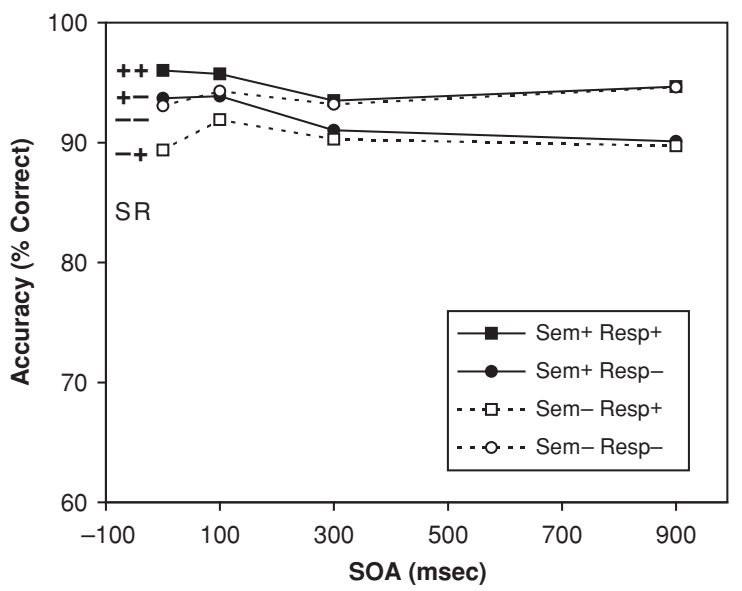

B Different Hands for T1 and T2 (Univalent)

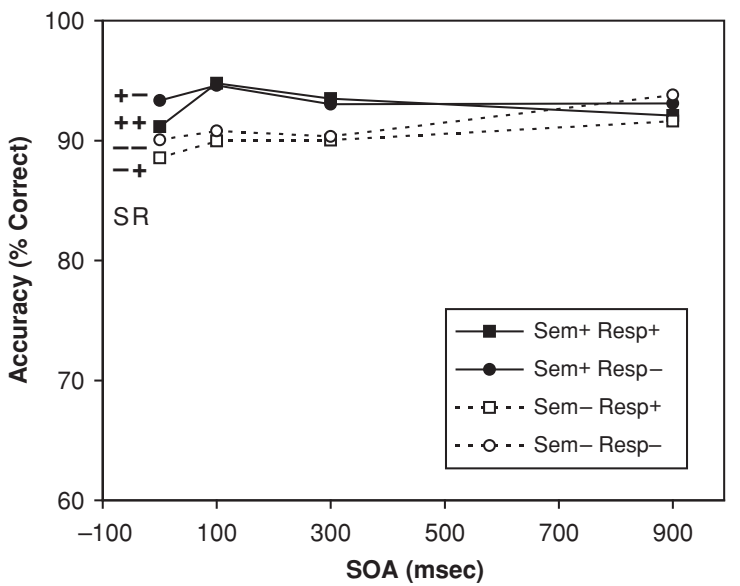

Figure 3. Experiment 1: Same-task-set mean combined accuracy for Tasks 1 and 2 (T1 and T2, respectively).

tic compatibility $[F(1,28)=17.17, p<.001]$ and SOA $[F(3,84)=4.51, p<.01]$, and the interaction of these factors was also significant $[F(3,84)=4.30, p<.01]$. A two-way interaction was also observed between SOA and single-/double-handed response condition $[F(3,84)=$ $3.74, p<.05]$.

It is important to note that the accuracy effects observed here essentially represent a summation of errors from Tasks 1 and 2, with the exception of those (assumedly few) trials in which errors occurred in both tasks. Informal inspection of individual trial data suggests that the large majority of errors were committed on Task 2, with relatively error-free Task 1 performance. As such, it is important to note that the accuracy effects of semantic and response compatibility shown in Figure 3 should not be misinterpreted as potential effects of Task 2 information on Task 1 performance. These accuracy measures appear to primarily (and probably only) reflect the effects of Task 1 information on Task 2 performance.

\section{Different-Task-Set Sessions}

Reaction time. Mean reaction time data for Tasks 1 and 2 in the different-task-set sessions are shown graphically in Figure 4. Again, the typical PRP effects were seen, with RT2 increasing with decreasing SOA, approaching a slope of -1 at the $0-\mathrm{msec}$ SOA. RT1 was relatively flat across SOAs. Set-switching costs were also observed, with same-task-set conditions faster than different-taskset conditions by an average of $112 \mathrm{msec}$ for Task 1 and 237 msec for Task 2 (also see Logan \& Gordon, 2001; Logan \& Schulkind, 2000).

For Task 1, semantic and response priming effects were observed at the 0-msec SOA for single-handed (bivalent) responses. The pattern of effects observed here was similar to that seen in the corresponding same-task-set data (above). Double-facilitation trials $(\mathrm{Sem}+\mathrm{Resp}+)$ were fastest, followed by double-interference trials (SemResp-), and then mixed-facilitation/interference trials $(\mathrm{Sem}-\mathrm{Resp}+$ and Sem + Resp -$)$. These effects were not observed at the $100-\mathrm{msec}, 300-\mathrm{msec}$, or $900-\mathrm{msec}$ SOAs for single-handed responses. The double-handed (univalent) responses were uniformly slower and did not show any semantic or response priming effects.

Inferential statistics confirmed the observations above for Task 1. There was a significant main effect of SOA $[F(3,90)=44.07, p<.001]$ but not of semantic or response compatibility. However, two-way interactions between semantic and response compatibility $[F(1,30)=$ 7.18, $p<.05]$ and between response compatibility and SOA $[F(3,90)=2.77, p<.05]$ were significant. Fisher's $\mathrm{LSD}_{.05}$ for the highest order interaction was $21.09 \mathrm{msec}$. By this criterion, the double-facilitation condition (Sem+ Resp+) was significantly faster than both of the mixedfacilitation/interference conditions (Sem + Resp - and Sem- Resp+) at the 0-msec SOA, but not at subsequent SOAs, for single-handed responses. The double-handed responses again showed no reliable pattern of differences.

The double-handed trials for Task 1 replicated the different-task-set findings of Logan and Schulkind (2000) and Logan and Gordon (2001), who used only doublehanded response mapping in their PRP tasks. The singlehanded response conditions were a new addition for the present experiments and revealed a new and important finding beyond those from previous studies. The finding here of Task 2 response information influencing Task 1 response selection suggests that Task 2 RS had begun before Task 1 RS was complete. This would appear to be a direct violation of the discrete-stage processing assumption.

For Task 2, semantic and response priming effects were observed at all SOAs for single-handed responses. The pattern of these effects was similar to that seen in Task 1 at the 0 -msec SOA and in the corresponding same-task-set 


\section{Same Hand for Task 1 and Task 2 Responses (Bivalent)}
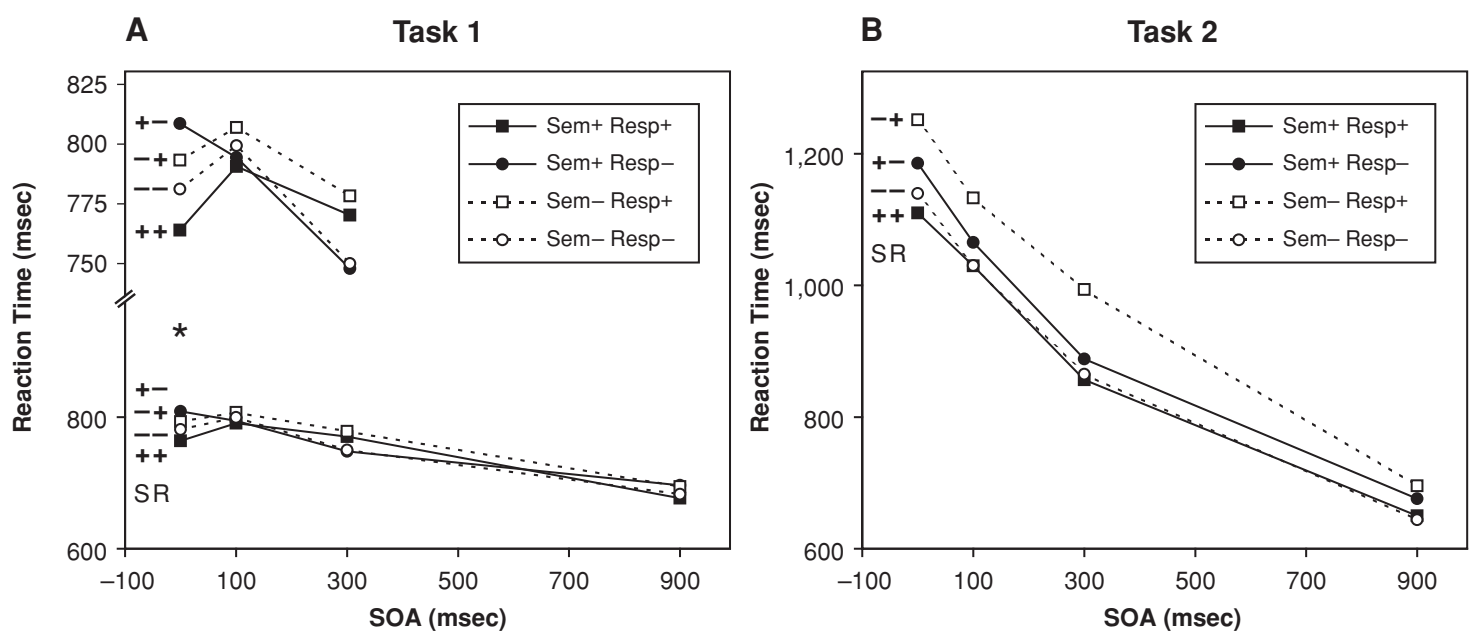

Different Hands for Task 1 and Task 2 Responses (Univalent)

C

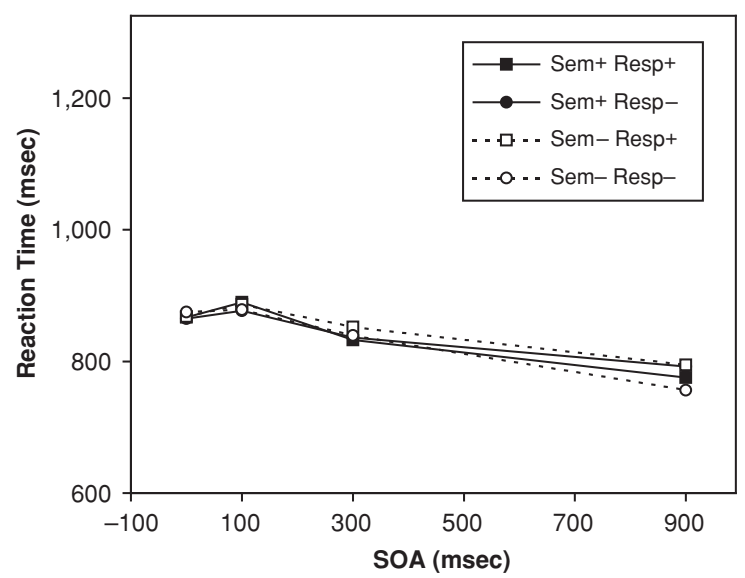

D

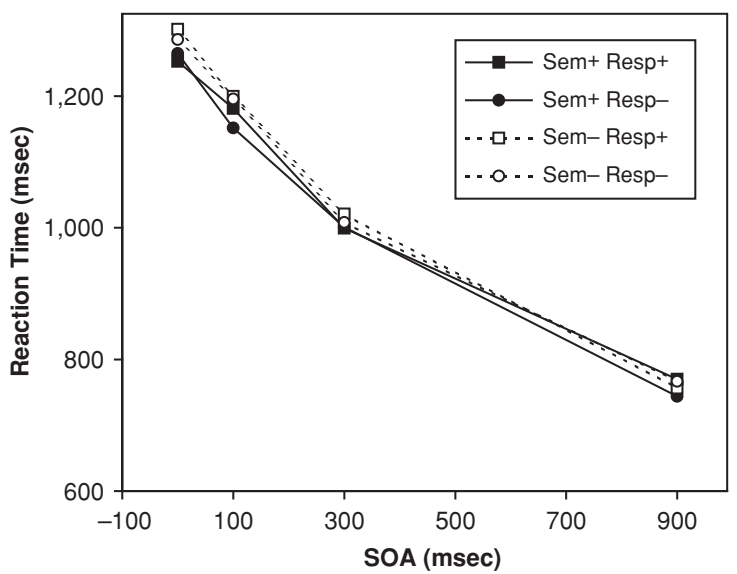

Figure 4. Experiment 1: Different-task-set mean reaction times for Tasks 1 and 2. The upper lines in panel A show a portion of the lower lines on a finer scale.

trials. The double-facilitation condition $(\mathrm{Sem}+\mathrm{Resp}+)$ was fastest, with the double-interference condition (SemResp-) following closely, appearing relatively slower only at the 0 -msec SOA. The semantic facilitation/response interference (Sem + Resp - ) and semantic interference/ response facilitation (Sem- Resp + ) conditions then followed in that order. In contrast, double-handed responses were uniformly slower and did not appear to be influenced by semantic or response compatibility.

These observations were supported by inferential statistics for Task 2. Main effects of semantic compatibility $[F(1,30)=22.97, p<.001]$, response compatibility $[F(1,30)=10.69, p<.01]$, and SOA $[F(3,90)=545.37$, $p<.001]$ were all significant. The two-way interactions between semantic and response compatibility $[F(1,30)=$ $11.27, p<.01]$ and between semantic compatibility and SOA $[F(3,90)=3.73, p<.05]$, as well as the three-way interaction between these factors $[F(3,90)=5.03, p<$ $.01]$, were also significant. A three-way interaction between semantic and response compatibility and single-/ double-handed response was also reliable $[F(1,30)=$ $12.99, p<.01]$. Fisher's LSD $_{.05}$ for the highest order interaction was $23.76 \mathrm{msec}$. By this criterion, the doublefacilitation condition (Sem + Resp + ) was significantly faster than both mixed-facilitation/interference conditions $(\mathrm{Sem}+\mathrm{Resp}-$ and Sem $-\mathrm{Resp}+)$ at all SOAs for singlehanded responses in Task 2. No such differences were observed for the double-handed conditions.

The double-handed trials for Task 2 again replicated the different-task-set findings of Logan and Schulkind (2000) and Logan and Gordon (2001). The new singlehanded conditions demonstrated effects of semantic and response compatibility of Task 1 information on Task 2 performance. Again, these Task 2 findings are not diag- 
nostic with regard to the main theoretical issue, the validity of the discrete-stage processing assumption. They may, however, provide additional reassurance with regard to Task 1 crosstalk findings, since the pattern of significant semantic and response effects on Task 2 was extremely consistent with the pattern of significant effects observed at the 0 -msec SOA in Task 1.

To try to better assess the crosstalk effects in different response conditions, the different-task-set data were reanalyzed with separate 2 (semantic compatibility) $\times 2$ (response compatibility) $\times 4$ (SOA) repeated measures ANOVAs for single- and double-handed response conditions in Tasks 1 and 2. As before, semantic compatibility, response compatibility, and SOA were all within-subjects factors.

The results were clear. The single-handed results were quite similar to those for the combined data. For Task 1, only the main effect of SOA was significant $[F(3,45)=$ 22.24, $p<.001$ ], but interactions between semantic and response compatibility $[F(1,15)=4.88, p<.05]$ and between response compatibility and SOA $[F(3,45)=4.95$, $p<.01]$ confirmed the observed effects of semantic and response priming at the shortest SOA. A single 2 (semantic compatibility) $\times 2$ (response compatibility) repeated measures ANOVA for single-handed responses at the 0 -msec SOA showed a significant interaction between semantic and response compatibility effects $[F(1,15)=$ $5.51, p<.05]$. Task 2 main effects of semantic compatibility $[F(1,15)=93.60, p<.001]$, response compatibility $[F(1,15)=11.58, p<.01]$, and SOA $[F(3,45)=210.14$, $p<.001]$ were all significant. The two-way interactions between semantic and response compatibility $[F(1,15)=$ 97.27, $p<.001]$ and semantic compatibility and SOA $[F(3,45)=4.16, p<.05]$, and the three-way interaction between these variables $[F(3,45)=6.74, p<.01]$, were also significant, confirming the observed effects on Task 2 over all SOAs for single-handed responses.
The double-handed response conditions were quite different. Only the main effect of SOA was significant for both Task $2[F(3,45)=378.73, p<.001]$ and Task 1 $[F(3,45)=21.97, p<.001]$. Other than a marginal effect of semantic compatibility observed for Task $2[F(1,15)=$ $3.47, p=.082]$, no semantic or response compatibility effects were observed at all.

Accuracy. Mean combined accuracy measures for Tasks 1 and 2 for different-task-set sessions are shown in Figure 5 for single- (bivalent) and double-handed (univalent) responses. Given the separation of semantic and response compatibility effects observed in the RT data, separate three-way ANOVAs were conducted for singleand double-handed response data, identical to those for mean reaction time above. Accuracy effects were again quite consistent with those for the reaction time measures. In general, higher mean error rates were observed in conditions with slower mean reaction times, with no speed-accuracy trade-off evident. Both semantic and response compatibility appeared to influence performance in both single- and double-handed response conditions, where similar effects on reaction time were observed only with single-handed responses. Notably, the sizes of the accuracy effects on single-handed responses were twice as large as those on double-handed responses. In addition, errors generally appeared to be more common as SOA decreased.

These observations were supported by inferential statistics. For double-handed responses, main effects of semantic compatibility $[F(1,15)=5.81, p<.05]$ and SOA $[F(3,45)=5.97, p<.01]$ were reliable, and the main effect of response compatibility was marginal $[F(1,15)=$ $3.10, p=.099]$. These effects were modified by a significant three-way interaction between the factors $[F(3,45)=$ $3.92, p<.05]$. For the single-handed response data, the main effect of response compatibility was reliable

\section{Overall Accuracy Given Correct Response on Both Tasks}
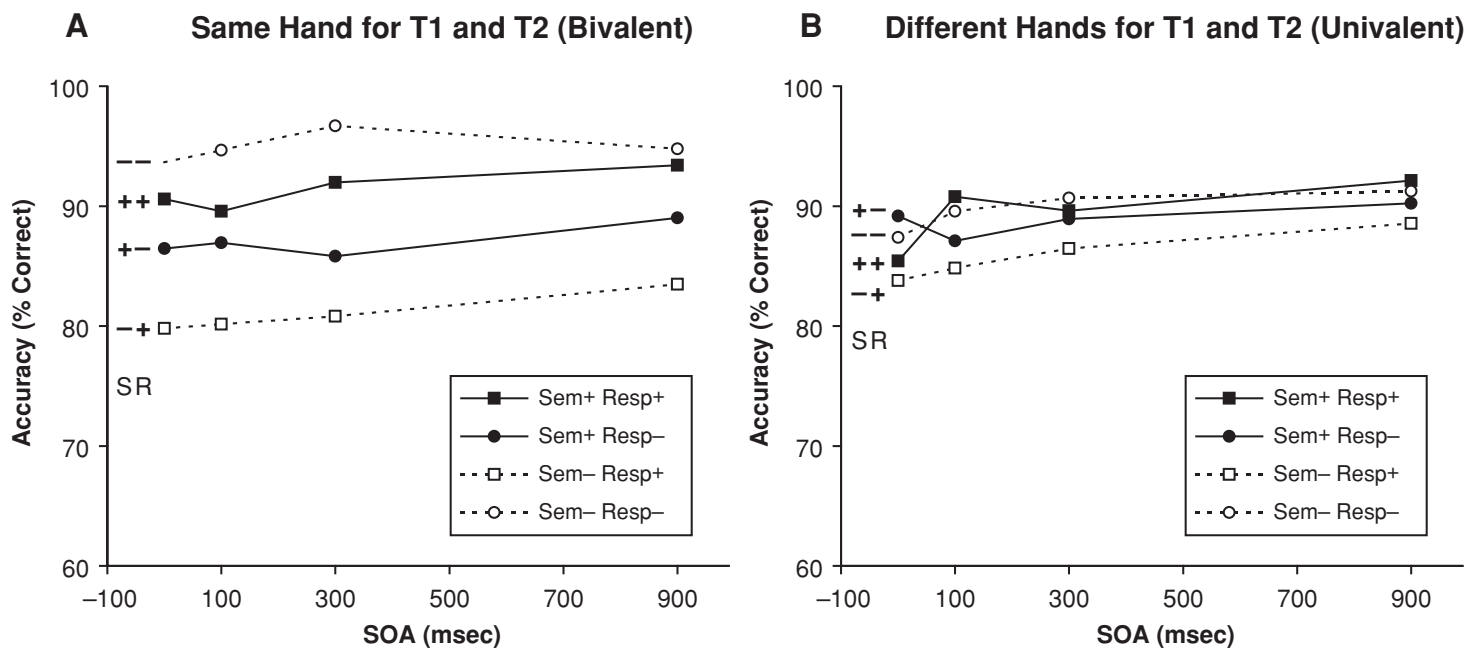

Figure 5. Experiment 1: Different-task-set mean combined accuracy for Tasks 1 and 2 (T1 and T2, respectively). 
$[F(1,15)=14.17, p<.01]$, as was the two-way interaction between it and semantic compatibility $[F(1,15)=$ $19.30, p<.01]$. In addition, the main effect of SOA was marginal $[F(3,45)=2.80, p=.051]$.

Again, it must be noted that the accuracy effects observed here essentially represent a summation of errors from Tasks 1 and 2, minus those trials where errors occurred in both tasks. Informal inspection of individual different-task-set trial data again suggested that the large majority of errors were committed on Task 2, with relatively error-free Task 1 performance. As such, it is important to note that the accuracy effects of semantic and response compatibility shown in Figure 5 should not be misinterpreted as potential effects of Task 2 information on Task 1 performance. These accuracy measures appear to primarily (and probably only) reflect the effects of Task 1 information on Task 2 performance.

\section{Discussion}

This experiment was designed to investigate the possibility of parallel response selection in a PRP paradigm. The cross-task influence of semantic and response information was investigated with regard to both Task 1-toTask 2 and Task 2-to-Task 1 priming effects. RSB theory relies on a discrete-stage processing assumption, which states that the RS processes of Tasks 1 and 2 must be discrete and serial with respect to each other - that is, Task 2 RS cannot begin until Task 1 RS is complete. RSB theory allows Task 1-to-Task 2 priming of RS information (as would be generally expected), but not the reverse.

Data from both same- and different-task-set conditions showed that Task 1 RS was influenced by Task 2 response information. In order for this to occur, Task 2 processing up to and including generation of substantial R2 information must have been active prior to the completion of Task 1 RS. Recall that this is in an experimental setting in which subjects were instructed to complete their response to Task 1 as quickly as possible, before attempting to perform Task 2.

The different-task-set conditions were particularly important here, because they allowed an independent withinsubjects assessment of response crosstalk effects that was orthogonal to potential semantic effects. The observation of Task 2-to-Task 1 response crosstalk at the 0-msec SOA in the single-handed (bivalent) response condition is the single most important finding here. The absence of these effects at later SOAs is in keeping with general assumptions about the time course of the tasks. It is straightforward to predict that as Task 1 processing progresses, it will at some point no longer be susceptible to task-related information. Task 2 information cannot be derived until $\mathrm{S} 2$ is presented, so one would predict that there should be some SOA cutoff where susceptible Task 1 processes have progressed too far to be influenced by Task 2 information. This assumption provides a useful check for our experiments, in that Task 1 should show compatibility effects from Task 2 information at short SOAs only (if they occur at all), and that such effects should be absent at longer SOAs. This was indeed the pattern of results observed throughout the Experiment 1 data, which increases our confidence in the Task 1 response compatibility effects observed.

One potential concern for the interpretation of our data involves the way in which subjects performed the two tasks within a single trial. In order to interpret effects of R2 information on Task 1 RS, we must assume that subjects are performing the two tasks in strict serial order and not attempting to perform Task 2 before they have responded to Task 1 . Obviously, computing the responses to both tasks and then making a combined double response for the whole trial could generate the response compatibility effects we observe with strict serial RS performance. Though we went to great pains to encourage subjects to perform the two tasks completely separately and serially, several aspects of the data also suggest that response grouping is unlikely to be the cause of the crosstalk effects observed here.

We consider our different-task-set RT data (Figure 4) to present the strongest case for parallel RS processing, and consider these data primarily. Here, the critical result in question is the compatibility effect in RT1 at the $0-\mathrm{msec}$ SOA for single-handed response conditions. Could the Task 1 compatibility effects we observed be due to subjects delaying $\mathrm{R} 1$ in order to make a grouped $\mathrm{R} 1+\mathrm{R} 2$ response, perhaps only on a subset of trials? If $R 1$ were delayed in this fashion, we might expect to observe a slower RT1 at the $0-\mathrm{msec}$ SOA than at the $100-\mathrm{msec}$ SOA, where no compatibility effects were observed. This was not the case, since RT1 was faster at the 0 -msec than at the 100msec SOA. Even if a subset of 0-msec-SOA trials were performed with a response-grouping strategy, and only these trials had Task 2-to-Task 1 influences, we would still expect the mean RT1 to be slower at the 0 -msec than at the 100-msec SOA, where no such effects were observed.

Several other factors argue against a response-grouping explanation. The random presentation order of differentSOA trials, combined with the imperative Task 1 performance requirements, would have made it extremely difficult for subjects to selectively perform 0 -msec-SOA trials with a response-grouping strategy without having this strategy influence trials at other SOAs, particularly $100 \mathrm{msec}$. Put another way, given the lack of compatibility effects in Task 1 at the 100-msec SOA (from response grouping or otherwise), it seems unlikely that subjects could selectively perform randomly intermixed 0 -msecSOA trials with a response-grouping strategy without incurring any kind of RT cost on Task 1 performance. The lack of RT1 compatibility effects at longer SOAs, the lack of such effects in any of the double-handed (univalent) response conditions (whose RTs were a good deal slower than in the bivalent conditions), as well as the consistent findings of characteristic PRP effects throughout the data set, also suggest that response-grouping strategies are not the cause of the crosstalk effects we observed.

\section{Extending Information Crosstalk Approaches}

Within our results, a number of important boundary conditions and other effects were identified. Responding 
with one hand for Task 1 and the other for Task 2 (univalent responding) was consistently slower in all conditions than was making both responses with the same hand (bivalent responding). This was an interesting and somewhat surprising finding, given that most PRP studies are conducted with different hands or response modalities (e.g., manual and vocal responses for Tasks 1 and 2, respectively) in order to minimize response conflict/interference, and supposedly to improve performance.

Logan and Schulkind's (2000) results were replicated here as the double-handed response condition data. Within this half of the design, the same parallel semantic retrieval effects they described (in addition to our parallel responseselection effects) were observed in same- but not in different-task-set trials (also see Logan \& Gordon, 2001). Logan and Schulkind suggested that parallel semantic memory retrieval was dependent on both tasks using the same task set. Logan and Gordon embodied this suggestion in a formal model of the PRP task that accounted for crosstalk between tasks. Although their suggestion was reasonable in light of their data, the present results suggest that it is not entirely the case. Both semantic retrieval and response-selection processes were seen to occur in parallel in same- and different-task-set conditions here, with data suggestive of parallel RS processing being dependent on responding to both tasks with the same hand. However, crosstalk effects were smaller when different task sets were used for the two tasks, compared with the sametask-set conditions. Logan and Gordon discussed these potential differences in regard to semantic crosstalk, noting that the same-task-set trials may be influenced by both the semantic compatibility of memory retrieval required for each task and flanker-like semantic compatibility effects (Eriksen \& Eriksen, 1974), but different-task-set trials may only be influenced by the latter.

The abolition of practically all cross-task semantic and response priming in different-task-set conditions with double-handed responses is also quite interesting and warrants further investigation. Considering hand and finger response mapping as part of a required task set, substantially greater reconfiguration would be required between tasks for double-handed than for single-handed differenttask-set trials, or for any of the same-task-set conditions. It is also possible that changing certain task set components (e.g., response mapping between hands) may impose a greater cognitive or time cost or may otherwise disrupt these effects. Such suggestions are only speculative, and further experimentation is needed to investigate these and related issues.

Finally, throughout our data, a recurring pattern of semantic and response compatibility interaction was observed. In general, where such interactions were observed - namely, at short SOAs for Task 1 and all SOAs for Task 2 in all same-task-set conditions and in the bivalent different-taskset conditions - the combined semantic- and responsecompatible condition (Sem + Resp + ) was fastest, followed by the combined semantic- and response-incompatible condition (Sem- Resp-), with the mixed-compatibility con- ditions (Sem + Resp - and Sem - Resp + ) slowest. Such an interaction is quite interesting, and it most basically suggests that semantic and response compatibility effects are not completely isolated and simply additive in their effects. The more substantial question is why these apparently separate effects should interact as we have observed.

A consideration of the overall degree of both semanticand response-related conflict or mismatch present in particular trial situations may provide a good starting point from which to consider these effects. Specifically, the combination of particular semantic and response compatibilities may produce conflict effects above and beyond basic effects of semantic and response compatibility/ facilitation alone. When the stimulus and response for the currently attended task are compatible with the semantic categorization of the other stimulus under the current task set, as well as with the motor response for the other task (i.e., the Sem + Resp + condition), there is no conflicting information. When the stimulus and response for the currently attended task are incompatible with the semantic categorization of the other stimulus under the current task set, as well as with the motor response for the other task (i.e., the Sem - Resp - condition), there is a presumed lack of facilitation, and our data show reaction times to be slower for this condition in most cases. However, we suggest that there may be relatively little conflict in this situation. There may be some benefit (perhaps more correctly, a lack of detriment) to having to make an incompatible motor response when an incompatible semantic flanker is present-essentially, that there is less inherent conflict in selecting the opposite motor response when there is a mismatch in semantic information. Note that in this situation, the relationship between the stimulus for the other task and the incompatible motor response in our experiment was not predictive under the attended task's task set; the relationship here was orthogonal by design.

Along these lines, the cases of highest conflict may be those conditions with mixed semantic and response compatibility, and not the dual-incompatible case. Consider the situation in which the stimulus for the currently attended task is incompatible with the semantic categorization of the other stimulus under the current task's task set, but the motor response required is compatible with that of the other task (the Sem - Resp + condition). In this situation, the semantic information may drive the selection of the correct compatible motor response, but the mismatch in semantic category may itself provide some signal favoring an opposite response, above and beyond the first-order semantic and response mappings inherent in the current task set. The more general point is that such mismatch signals may add additional conflict or other noise to the system, necessitating greater protection of these processes and resulting in longer reaction times and higher error rates, as observed in our data. The effects of the other mixed-compatibility condition $(\mathrm{Sem}+\mathrm{Resp}-$ ) can be considered in a similar way.

Most importantly, it should be noted that with or without such interactions, the primary issue is whether we 
can detect the influence of Task 2 response information on Task 1 response selection. We suggest that in order to generate such R2-to-R1 effects (independent of S2-toR1 effects), whether they interact with other factors or not, requires R2 information to be generated prior to the completion of Task $1 \mathrm{RS}$, which is the principal issue at stake here.

Similar effects have been observed previously, most recently in the literature on task set switching. For example, Rogers and Monsell (1995) found similar interactions with response repetition effects and set switching in five experiments using an alternating-runs paradigm. When the current trial required the same task to be performed as on the previous trial (no switch of task set), performance was faster when the same motor response was required as on the previous trial, compared with making the alternate motor response - a typical response repetition facilitation effect. However, when the current trial required a different task to be performed than on the previous trial (a task set switch), performance was slower when the same motor response was required as on the previous trial, compared with making the alternate motor response. Our interactions of semantic and response compatibility effects closely mirror these findings from the set switching literature. Rogers and Monsell suggested several potential mechanisms for such effects, some of which are similar to those we describe above, including both prevention of response perseveration and the increased requirements of control processes to prevent error in the face of greater informational mismatch. Though these ideas are quite speculative, this particular pattern of semantic and response interaction appears to be robust, and as we discussed above, these priming results are unlikely to be due to response grouping, reversal of task performance order on a subset of trials, or other similar strategic delaying in performance. These priming effects and the interesting interactions we observed here appear to be real, and they warrant more study.

\section{EXPERIMENT 2}

The first experiment demonstrated that Task 1 response selection could be influenced by information arising from Task 2 RS processes, within an experimental paradigm that stressed strict serial Task 1-then-Task 2 performance of the two tasks. These effects were observed with both single- (bivalent) and double-handed (univalent) response conditions when the same task was performed for Tasks 1 and 2, but such effects were present only when the same hand was used to respond to different tasks for Tasks 1 and 2 .

The second experiment sought to replicate the key findings of Experiment 1-namely, the ability for Task 2 $\mathrm{RS}$ information to influence Task $1 \mathrm{RS}$. In addition, we sought to test the limits of the apparent boundary condition of the effect, requiring within-hand (bivalent) motor response mapping for both tasks. For this experiment, we used only the most critical quarter of the design from Experiment 1-different tasks for Task 1 and Task 2 with repeated (equivalently bivalent) response mapping. Instead of mapping responses for both tasks to the index and middle fingers of the same hand, we mapped response alternatives for each task to the right or the left index finger for both Tasks 1 and 2. We hypothesized that we would observe the same kind of effects as in the single-handed response conditions in the different-task-set sessions in Experiment 1 -effects of semantic and response compatibility at all SOAs in Task 2 and, critically, the same pattern of effects at the shortest SOA(s) in Task 1.

\section{Method}

\section{Subjects}

Sixteen undergraduates from the University of Illinois at Urbana-Champaign participated in a single $1-\mathrm{h}$ session. The subjects received either partial course credit or $\$ 6 / \mathrm{h}$ for their participation. All subjects were right-handed and had normal or corrected-to-normal vision.

\section{Apparatus and Stimuli}

The stimuli and computer apparatus were the same as for Experiment 1. Responses were collected using the "." and " $x$ " keys of the computer keyboard. The stimuli were presented as in Experiment 1, with the same SOAs and event timing.

Task 1 and Task 2 were always different in this experiment. Half of the subjects performed the parity task ("Is S1 odd or even?") as Task 1 and the magnitude task ("Is S2 higher or lower than 5?") as Task 2. The other half performed the magnitude task as Task 1 and the parity task as Task 2 .

\section{Procedure}

The experimental design involved 512 trials, formed from two replications of the basic 256 -trial iteration ( 8 digits for $\mathrm{S} 1 \times 8$ digits for S2 $\times 4$ SOAs), in randomized order and presented in blocks of 64 trials interleaved with rest periods, as in Experiment 1. Prior to each 512-trial experimental session, the subjects performed an initial practice block of 64 trials, which was not considered for analysis.

The subjects indicated their responses to the stimuli by pressing the "." and " $x$ " keys of the computer keyboard with the index fingers of their right and left hands, respectively. All subjects made responses to both tasks with the same effectors - right versus left index fingers for Task 1, and then again for Task 2. Mapping of semantic classification (odd/even and high/low) to right/left motor response was constant for individual subjects throughout the experiment and was completely counterbalanced between subjects.

The subjects received the same instructions as for Experiment 1. They were instructed to respond as quickly and as accurately as they could, with Task 1 pertinence and serial performance of the tasks emphasized at length. The subjects were told to concentrate on S1 for each trial and to make their response to it as quickly and as accurately as they could. Only once they had responded to S1 should they then think about $\mathrm{S} 2$ and try to respond quickly and accurately to it. The subjects were explicitly told not to wait until they knew both answers or to make a joint response, but to think of each trial as two completely separate tasks to be done in strict order.

\section{Results}

\section{Reaction Time}

Mean reaction times for Tasks 1 and 2 were computed from trials in which responses to both tasks were correct. Data from 2 subjects were excluded from analysis because of mean reaction times more than two standard deviations slower than those of the other subjects, as well as error rates more than twice that of all other subjects. The anal- 
yses here concern data from the 14 remaining subjects. Analyses of reaction time measures are presented first, followed by an analysis of the overall and task-relative accuracy measures.

Mean reaction time data for Tasks 1 and 2 are shown graphically in Figure 6. Separate 2 (semantic compatibility) $\times 2$ (response compatibility) $\times 4(\mathrm{SOA})$ repeated measures ANOVAs were conducted for Task 1 and Task 2 mean reaction time data, with all factors within subjects. As throughout Experiment 1, the typical PRP effects were observed, with RT2 increasing with decreasing SOA, approaching a slope of -1 at the 0 -msec SOA, and RT1 remaining relatively constant across SOAs.

For Task 1, semantic and response compatibility effects were observed at the $0-\mathrm{msec}$ SOA and not at later SOAs, closely matching the effects seen in the corresponding conditions (different task sets with single-handed response mapping) of Experiment 1. Double-facilitation trials $(\mathrm{Sem}+\mathrm{Resp}+)$ were fastest, followed by doubleinterference trials (Sem- Resp-), and then mixedfacilitation/interference trials (Sem- Resp + and Sem + Resp-). This pattern was also suggested by the data at the 100-msec SOA, but the magnitude of differences between conditions was much smaller here.

These observations were supported by inferential statistics, with a significant interaction observed between semantic and response compatibility $[F(1,13)=6.35, p<$ $.05]$. The main effect of SOA was also reliable $[F(3,39)=$ $21.89, p<.001]$. An additional 2 (semantic compatibility) $\times 2$ (response compatibility) repeated measures ANOVA for the 0-msec-SOA data showed a significant interaction between semantic and response compatibility effects $[F(1,13)=5.61, p<.05]$.

The response compatibility effect of Task 2 response information on Task 1 performance observed at the 0 -msec SOA replicated the critical finding in the single-handed (bivalent) response conditions of the different-task-set sessions of Experiment 1. This finding suggests that Task 2 RS was active prior to the completion of Task $1 \mathrm{RS}$, in violation of the discrete-stage processing assumption.

For Task 2, semantic and response compatibility effects were observed, closely matching the effects at the 0 -msec SOA in Task 1, as well as those seen in the corresponding conditions of Experiment 1. Across SOAs, the doublefacilitation $(\mathrm{Sem}+\mathrm{Resp}+)$ condition was fastest; the double-interference (Sem- Resp-) condition followed closely, and even appeared to be somewhat faster at longer SOAs. The semantic facilitation/response interference $(\mathrm{Sem}+\mathrm{Resp}-)$ and semantic interference/response facilitation (Sem-Resp+) conditions then followed in that order.

Inferential statistics supported these observations. Significant main effects were observed for response compatibility $[F(1,13)=4.96, p<.05]$ and SOA $[F(3,39)=$ $211.58, p<.001]$. Significant two-way interactions were observed for semantic and response compatibility $[F(1,13)=32.76, p<.001]$ and for semantic compatibility and SOA $[F(3,39)=3.05, p<.05]$. The three-way interaction between these factors was marginal $[F(3,39)=$ 2.24, $p=.098]$.

Task 2 crosstalk findings replicated those of the equivalent conditions in Experiment 1. It should again be noted that these Task 1-to-Task 2 crosstalk effects are not diagnostic in regard to the validity of the discrete-stage processing assumption. They may, however, provide additional confidence in the Task 1 crosstalk results, with regard to the similarity of patterns of semantic and response compatibility effects observed in both tasks.

\section{Accuracy}

Mean combined accuracy measures for Tasks 1 and 2 (again classified with regard to Task 1 semantic and response categories) are shown in Figure 7. These data again

\section{Both Hands for Each Task 1 and Task 2 Response}
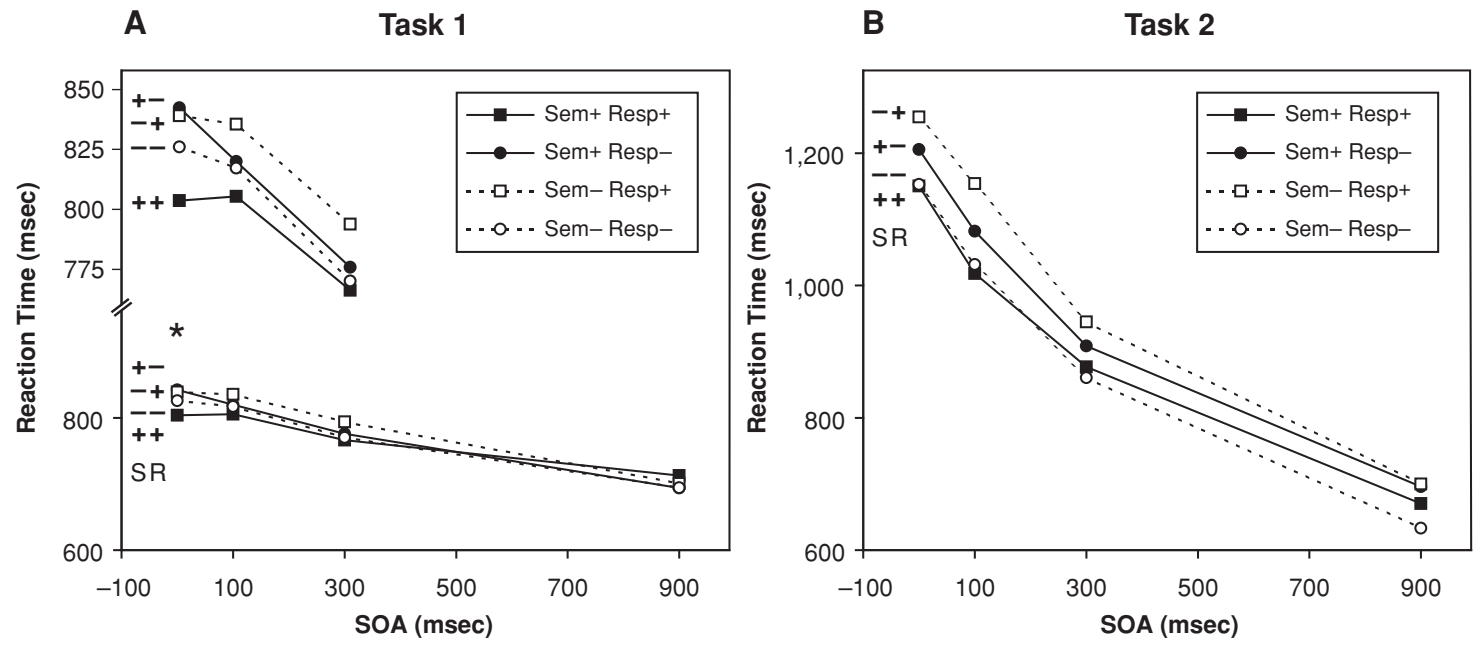

Figure 6. Experiment 2: Mean reaction times for Tasks 1 and 2 (different task sets). The upper lines in panel A show a portion of the lower lines on a finer scale. 
represent the combined errors from both tasks, since an error in either task disqualified an entire trial from consideration for RT analysis. These data were quite similar to those from the analogous conditions in Experiment 1 (different task sets with single-handed [bivalent] response mapping; see Figure 5A).

Given our informal observation in Experiment 1 that most errors arose from Task 2, we rescored our raw data from Experiment 2 to separately assess errors committed on Task 1 and on Task 2, given a correct response in the other task. These mean accuracy data for Task 1 and Task 2 separately are presented in Figure 8. Consistent with our informal observations in Experiment 1, the large majority of errors were observed in Task 2, with relatively error-free performance in Task 1 (lowest mean accuracy for Task 1 was $97.2 \%$, for the Sem + Resp - condition at the 900-msec SOA). Task 2 accuracy effects were consistent with those for the reaction time measures, with higher mean error rates generally observed in conditions with slower mean reaction times, suggesting an underlying process driving both rather than a speed-accuracy trade-off.

Separate three-way repeated measures ANOVAs were conducted for the Task 1 and Task 2 mean accuracy data, exactly as for the mean reaction time measures. Inferential statistics supported the observations above. For Task 2, the main effect of response compatibility was significant $[F(1,13)=17.11, p<.01]$, with a marginal main effect of semantic compatibility $[F(1,13)=4.31, p=.058]$; both were modified by a significant two-way interaction $[F(1,13)=22.87, p<.001]$. In addition, the two-way interaction of semantic compatibility and SOA was marginal $[F(3,39)=2.71, p=.058]$.

For Task 1, only a main effect of semantic compatibility was evident $[F(1,13)=5.67, p<.05]$, with a marginal two-way interaction of response compatibility and SOA $[F(3,39)=2.47, p=.076]$. Given our focus on the se-

(Correct Responses or
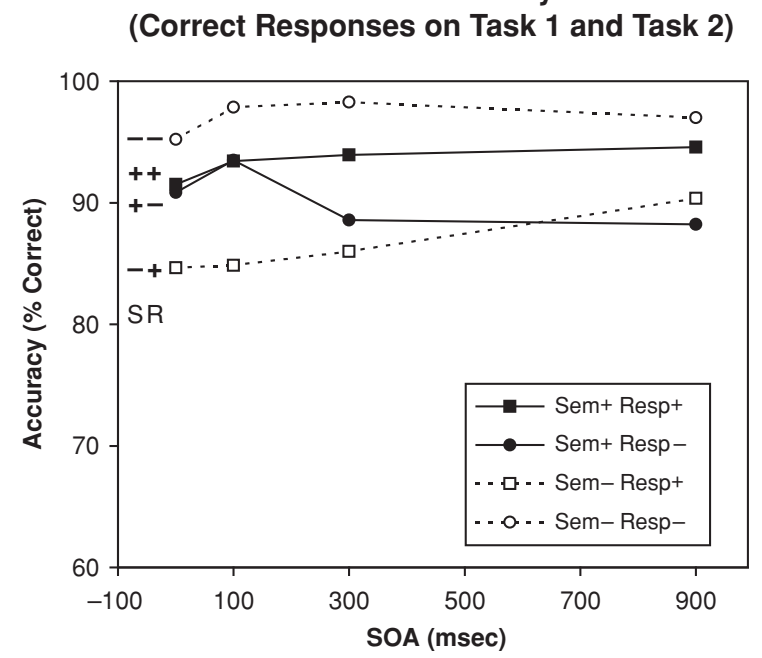

Figure 7. Experiment 2: Mean combined task accuracy (different task sets). mantic and response effects on RTs at the 0-msec SOA for Task 1, we performed an additional 2 (semantic compatibility) $\times 2$ (response compatibility) repeated measures ANOVA to assess specific accuracy effects here. A significant main effect of semantic compatibility was observed again $[F(1,13)=5.45, p<.05]$, but no effect of response compatibility was found, nor any interaction of the two factors.

\section{Discussion}

Results from Experiment 2 closely mirrored those from the equivalent conditions of Experiment 1, with different task sets for Tasks 1 and 2 and bivalent response mapping. A now-characteristic pattern of response and semantic compatibility effects was observed at all SOAs for Task 2 and, critically, at only the shortest SOA for Task 1 . These data replicate the findings of Experiment 1 and again suggest that Task 2 RS information was available prior to the completion of Task $1 \mathrm{RS}$.

The alternative bivalent response mapping used for this task (right vs. left hand response alternatives for both Tasks 1 and 2) did not disrupt response or semantic crosstalk effects, as the different-handed (univalent) responses did for different-task-set performance in Experiment 1. This contrast suggests some functional equivalence between the bivalent response methods used in the two experiments here and may be an interesting issue for further investigation.

\section{GENERAL DISCUSSION}

This article investigated whether the response-selection processes of both tasks of a dual-task pair could operate in parallel in a typical PRP paradigm. Specifically, we sought to test the discrete-stage processing assumption, which RSB theory depends upon in interpreting RT data to identify the bottleneck. The locus-of-slack logic that underlies this interpretation assumes that the processes involved in the bottleneck-for RSB theory, the response-selection stage for both tasks in a PRP paradigm - are discrete and serial. As such, RSB theory suggests that Task 2 response selection cannot begin until Task 1 response selection is complete.

The basic design of our experiments attempted to independently assess how R1 was influenced not only by S2, but also by R2. The tasks were designed so that potential response compatibility effects would be completely orthogonal to potential semantic compatibility effects. The different-task-set sessions enabled a more controlled analysis, removing the potential for semantic effects due to a common task set being used for both tasks. Under the different-task-set arrangement, $\mathrm{S} 2$ has its effect on R1 by influencing the semantic classification of S1 under the Task 1 task set-essentially a flanker effect (Eriksen \& Eriksen, 1974). Response compatibility effects of (eventual) R2 on R1 may occur if a substantial amount of Task 2 $\mathrm{RS}$ information is available prior to the completion of Task 1 RS. In order for it to elicit Task 2 response infor- 


\section{Accuracy Given Correct Response on Other Task}
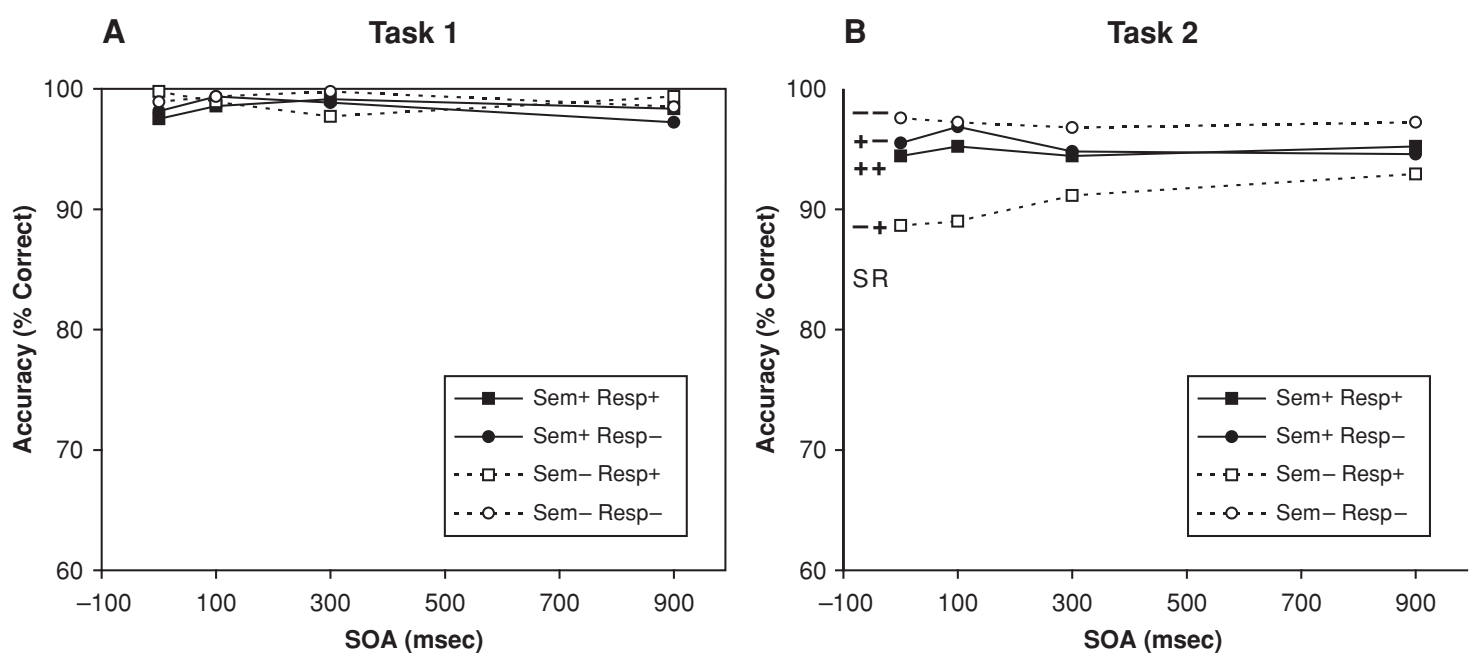

Figure 8. Experiment 2: Task-specific mean accuracy for Tasks 1 and 2 (different task sets).

mation (and influence the selection of the motor response for Task 1), S2 must be classified under the Task 2 task set prior to the completion of Task 1 RS.

Data from both experiments demonstrated cross-task influences of semantic and response information through both Task 1-to-Task 2 and Task 2-to-Task 1 priming effects. These effects were observed at all SOAs for Task 2 and, critically, at only short SOAs for Task 1 . This suggests that at short SOAs, Task 2 processing had progressed sufficiently to produce R2 information that could prime Task 1 RS. Priming of Task 1 RS from R2 information requires that Task 2 response processes begin before Task 1 RS is complete. Such findings suggest that Task 1 and Task 2 RS processes may operate in parallel. This would constitute a violation of the discrete-stage processing assumption, a key component of the locus-of-slack logic upon which RSB theory is based.

It is possible that RSB theory itself could be compromised because of this fundamental assumption. Whether or not RSB is affected by this challenge depends on the outcome of the mathematical analysis necessary to develop locus-of-slack predictions without assuming discrete stages (i.e., assuming cascaded stages). It is possible that all the predictions about additive and underadditive interactions with SOA would remain the same, in which case our present results would not challenge RSB theory. It is also possible that the predicted interactions will indeed change, in which case our results do challenge RSB theory. Ashby's (1982) and McClelland's (1979) analyses of single-task cascade processes showed that the patterns of interaction and additivity that cascaded stages predict are often different from the patterns that discrete stages predict. By induction, one might expect similar complications when the locus-of-slack predictions for cascaded stages are computed. At the very least, our work motivates further research into locus-of-slack predictions for cascaded processes or for other processes that do not assume discrete stages. It should be noted that our data in no way suggest a problem with the locus-of-slack logic itself. Indeed, this logic is extremely robust and useful, and we have no concerns with it at all. Our challenge is that it may not be a suitable model for characterizing PRP performance (as embodied in RSB theory), given that our data suggest parallel operation of RS processes.

\section{The Nature of the Bottleneck}

Although our data suggest the parallel operation of response-selection processes, for decades research with the PRP paradigm using locus-of-slack methods has shown remarkably consistent results, the large majority of which point toward a bottleneck in RS (but see Logan \& Gordon, 2001; Meyer \& Kieras, 1997a, 1997b; Navon \& Miller, 2002; Tombu \& Jolicœur, 2003). Might our data be accounted for within the current framework of RSB theory, which assumes that RS is serial and discrete?

One approach to this question (frequently suggested to the authors) concerns whether a single RS mechanism is being influenced by both stimuli to produce these results, or whether two separate mechanisms operating in parallel, RS1 and RS2, are necessary, with the momentary output of RS2 influencing RS1. The former conception has many variants, but the core model is as follows: ${ }^{1} \mathrm{~A}$ single RS mechanism exists and is employed to produce a response for Task 1. S2 information is extracted and processed via this mechanism along with S1. S2 affects RT1 by speeding/slowing RS for S1 when it is congruent/ incongruent with the informational relationships between S1 and R1. A more specific model might even specify separate potential compatibility/priming effects for S2, depending on (1) the relations between S1 and a target semantic classification/retrieval, and (2) either the relations between S1 and R1 or the semantic category of S1 and 
R1. Under any and all of these models, the effects of S2 on RT1 do not necessitate or imply the computation of R2. The key issue in this family of models is that S2 can have multiple effects, either via S2-S1 semantic effects, or via more direct S2-R1 compatibility effects due to overlap of stimulus and response sets of the two tasks, without having to implicate a second independent RS mechanism.

With this said, we suggest that our data do at least necessitate a model that allows for the separate and independent derivation of response information for two different tasks in parallel - that is, that S1 can be processed to produce R1 information via Task 1 task set rules, with $\mathrm{S} 2$ being processed in parallel to produce $\mathrm{R} 2$ information via Task 2 task set rules. The key issue lies in the very specific way we designed our tasks, which makes these experiments crucially different and more revealing than those of Hommel (1998), Lien and Proctor (2000), Logan and Schulkind (2000), Logan and Delheimer (2001), and Logan and Gordon (2001). Indeed, it is this very issue that represents the true test of RSB theory's discreteness assumption. In our experiments with different tasks for Tasks 1 and 2, R2, the correct motor response for S2 processed under the Task 2 task set, was completely orthogonal to the motor response generated if S2 was processed under the Task 1 task set (i.e., if S2 information was used to generate/influence R1).

Put another way, in our different-task-set conditions, the relationships of S1 to R1 and S2 to R1 were both determined by the Task 1 task set and were orthogonal to the relationship of S2 to R2 (determined by the Task 2 task set). As such, we were able to measure the effects of S2 on $\mathrm{R} 1$ separately and independently from the effects of R2 on R1. Thus, if we observed an effect of R2 on R1, we could conclude that it was not due to the influence of S2 on R1. The critical response compatibility measures we used in our study specifically assessed the relationship between $\mathrm{R} 2$ and $\mathrm{R} 1$. Finding consistent, systematic effects of R2 on $\mathrm{R} 1$ in this experimental context strongly suggests that R2 information was indeed generated prior to the completion of Task 1 RS. The only way to derive this R2 information from the given stimuli and tasks was to transform S2 via the Task 2 task set rules to generate R2 information. We argue that the process of transforming a stimulus under its own task set to generate response-related information is indeed response selection, and that this process is occurring for Task 2 in parallel with Task 1 to an extent sufficient to generate adequate $\mathrm{R} 2$ information to prime Task 1 response selection.

Another alternative explanation for our results (also often suggested to the authors) is that response-selection processes are indeed discrete and serial, and that our data instead reflect the priming of Task 1 response execution from Task 2 RS. From our design, we cannot rule out this possibility, although several factors suggest that this explanation is less plausible than the others we suggest here.

Coles, Gratton, Bashore, Eriksen, and Donchin (1985) used a single-task flanker paradigm (Eriksen \& Eriksen, $1974)$ and measured both electromyogram (EMG) and dynamometer squeeze activity for manual responses to assess the time course of human information processing. They observed that when incorrect squeeze activity was present, the time taken to execute the correct response was increased, supporting a notion of response competition. Within their analyses, they were able to separately assess the influence of noise/compatibility (i.e., flanker) effects on response-activation and response-execution processes. If one identifies response execution with the interval between the onset of EMG activity and the detection of an overt squeeze response, several pertinent findings may be noted. First, Coles et al. demonstrated that the interval between EMG onset and squeeze onset in the correct response channel was longer for arrays with incompatible flankers $(67 \mathrm{msec})$ than for arrays with compatible flankers $(59 \mathrm{msec})$, when other response-activation factors were controlled for. Adjusting this difference through weighting of condition means, they estimated a $12-\mathrm{msec}$ compatibility effect, separate from other, larger effects on response-activation processes, that was essentially due to the direct facilitation or priming of response-execution processes from peripheral information.

Although these data suggest that priming of responseexecution processes from response-activation/-selection processes is indeed possible, considering these data with respect to the effects we have demonstrated suggests that priming of Task 1 response execution from Task 2 RS information is unlikely to account for our effects. First, the average effect size of $12 \mathrm{msec}$ that Coles et al. (1985) observed for the flanker compatibility effect of response execution is quite small compared with the critical Task 2-to-Task 1 response compatibility effects we demonstrated here, which are in the range of $30-40 \mathrm{msec}$ for our most stringently controlled cases (the bivalent-response, different-task-set conditions) and are several times this size in other conditions. In addition, the 60- to 70-msec overall duration of the response-execution process as assessed by Coles et al. is in some cases shorter than the size of the compatibility effects we observed in our data, again suggesting that our effects cannot be well accounted for via priming of Task 1 response execution from Task 2 response selection.

A separate piece of evidence supporting our claim that Task 2 response information influences Task 1 response selection, not Task 1 response execution, is our finding of the interaction between semantic- and response-related information. The fact that these factors interact suggests that they operate at the same locus, rather than at sequential loci. There is a range of evidence suggesting that semantic effects operate in the evidence accumulation phase of response selection (for example, see Logan, 1980; Hommel, 1998), and this account is consistent with various randomwalk accounts of response selection (for a discussion, see Logan \& Gordon, 2001). If this is the case, and if interactions suggest that our semantic and response compatibility effects have the same locus, then the influence of Task 2 response information we observed on Task 1 would seem likely to reflect effects on Task 1 response-selection rather than response-execution processes. It should also be noted that a significant motivation for assuming a response ex- 
ecution priming account of these and similar data is to preserve the discrete-stage processing assumption of RSB theory. Though we cannot fundamentally disprove all potential accounts of Task 2 response information influencing Task 1 response execution as the cause of our results, we do suggest that an account including the priming of Task 1 response selection from Task 2 response information is a more plausible one.

\section{Alternative Models of Dual-Task Performance}

Given that we believe our evidence for the parallel operation of response-selection processes is substantial, what now might be done to reconcile this view with overt behavior (the PRP effect itself being a case in point), which consistently demonstrates large and persistent delays in Task 2 processing, despite an apparent parallel flow of information through the system for the two tasks? Several authors have begun to consider these and related issues.

One suggestion is that, though RS processes of both tasks may run in parallel, the amount of information derived from the unattended task (initially Task 2 ) is quite small relative to that from the attended task (initially Task 1). While subjects perform Task 1, enough Task 2 processing occurs to generate sufficient R2 information to influence R1 at short SOAs, but the majority of Task 2 RS is still incomplete when Task 1 RS finishes. As SOA increases, RS processes of the two tasks overlap less, and Task $2 \mathrm{RS}$ is attended to earlier (relative to presentation of $\mathrm{S} 2$ ), resulting in a quicker RT2 as SOA increases. This is similar to the RSB conception, except with limited rather than no RS processing for Task 2 during the "slack" period. However, an important caveat here relates to both the quantity and quality of Task 2 response information potentially generated in parallel with Task 1 RS. If relatively little R2 information were generated during Task $1 \mathrm{RS}$, or if this Task 2 information were particularly error-full, it could potentially influence Task 1 RS processes without being useful in the subsequent computation of R2 once Task 2 is attended. In such a situation, Task 2 RS would be considered informationally and functionally discrete from Task $1 \mathrm{RS}$, preserving the essential nature of the RS bottleneck, despite evidence (such as our data here) of R2 information influencing R1 behavior.

Most recently, Navon and Miller (2002) and Tombu and Jolicœur (2003) demonstrated that a resource model can account for typical PRP effects, if one assumes an asymmetric allocation of resources to the two tasks. They suggested that such an assumption was quite reasonable, given that the demand characteristics of PRP-like paradigms themselves are inherently asymmetric. A more substantial departure from these views is embodied in Meyer and Kieras's (1997a, 1997b) EPIC model. This view suggests that central capacity is essentially unlimited, and that serial task performance in dual-task situations is strategic, because of subjects' typical use of conservative or cautious performance strategies. EPIC has been shown to account for a substantial range of dual-task data, including bottleneck-like PRP effects, with a parallel-processing model.
Logan and Gordon (2001) also considered the nature of dual-task processing with their executive control theory of visual attention (ECTVA) model and provided formal and quantitative analyses of serial versus parallel performance of same- and different-task-set PRP task pairs almost identical to those in the experiments conducted here. The core machinery of ECTVA combines Bundesen's (1990) theory of visual attention (TVA) with an exemplar-based random-walk process (Nosofsky \& Palmeri, 1997) to model the accumulation of response-salient perceptual information, modulated by task-specific biasing of salient perceptual, response, and goal-based parameters. Logan and Gordon's simulations of PRP performance demonstrated that TVA produced faster PRP performance when run in series for the two tasks than when run in parallel. This serial performance of tasks is strategic rather than obligatory in ECTVA and is used to reduce interference from information generated in parallel from the other task, thus reducing the number of random-walk steps necessary to reach criterion for a response (equivalent to an RT measure). This is more reminiscent of Meyer and Kieras's (1997a, 1997b) EPIC model, with strategic serial behavior to protect performance, as opposed to RSB's obligatory serial nature.

Logan and Gordon's (2001) ECTVA model was used to produce data to model several PRP experiments quite similar to those conducted here. ECTVA was able to account for the crosstalk effects observed in their subject data, which were equivalent to the semantic crosstalk effects demonstrated in our present experiments. Logan and Gordon did not assess the potential for their ECTVA model to account for parallel response selection separately from semantic crosstalk effects, and the modification of their model to account for these data is beyond the scope of this article. With this said, the processing architecture we outline in Figure 1 and our data suggesting the necessity for separate simultaneous RS processes suggest several possibilities.

ECTVA's preference for performing tasks in series rather than in parallel is based upon simulations in which parallel performance assumes equal attentional bias/processing gain allocated to each task. The result is a substantial increase in the number of random-walk steps required to reach criterion (equivalent to RT) in parallel performance, because of excessive interference via semantic crosstalk from the concurrent task. If task performance were parallel but substantially biased toward one task at a time (i.e., initially biased to Task 1 in our experimental design), performance of that task could be protected from undue interference, yet information from the other task would also be allowed to be present, as our new data suggest is the case. Such a situation is not dissimilar to the biased parallel model described above. The modification of ECTVA to run as a biased parallel model would allow response information to be generated in parallel under both Task 1 and Task 2 task sets (although at quite different rates), making R2 information available to influence the exemplar-based random-walk process prior to the completion of $\mathrm{R} 1 \mathrm{selec}-$ tion. Such a model would predict that response informa- 
tion must be sufficiently compatible at a response level to contribute to the accumulation of response information in the random-walk process. Our present data support this prediction, since we observed response crosstalk effects only when the same motor responses were used for both tasks, not when corresponding motor responses in opposite hands were used.

In modeling PRP performance, Logan and Gordon's (2001) ECTVA model takes an inherently parallel, cascaded processing architecture and runs it in serial order, closely approximating the behavior of experimental subjects. As we did in the discussion above, one might ask whether key aspects of the model must necessarily operate serially to approximate subjects' behavior, or whether such behavior can be achieved with less extreme assumptions. One particular aspect that Logan and Gordon introduced to ECTVA (discussed further in Logan \& Delheimer, 2001) was the concept of "flushing" the response-selection machinery between performing Task 1 and Task 2 . Such flushing was achieved by resetting the random-walk counters to some fraction of their previous states, in order to prevent perseveration of the just-selected response, R1, and enable accurate selection of R2. In addition to protecting from perseverative behavior resulting from persistent R1 information influencing R2, such a flushing mechanism would also prevent any R2 information that had accumulated during Task 1 performance from influencing subsequent Task 2 response selection.

In this context, an important question with regard to our research is the extent to which this resetting of response-selection processes occurs. If flushing is complete (or close enough to it), response selection might be considered to be discrete, in that none of the R2 information accumulated during Task 1 performance would contribute to response selection of Task 2. Such a conception could potentially account for our present response crosstalk evidence showing R2 information influencing R1 at short SOAs, while maintaining informationally discrete RS processes apparently compatible with RSB theory.

A key issue, however, is just how much flushing is necessary to prevent response perseveration? Logan and Delheimer (2001) presented a discussion of this very issue, based partly upon the degree to which Logan and Gordon's (2001) ECTVA model required flushing of random-walk counter information to prevent response perseveration. By default in ECTVA, flushing is not complete, with counters reset to $10 \%$ of their final response-selection value after $\mathrm{R} 1$ is selected, in preparation for accumulating evidence to select R2. The degree of flushing necessary to prevent response perseveration in ECTVA is much smaller than the $90 \%$ figure used and is equivalent to the difference between counters being reset to a value slightly less than the criterion value (Logan \& Delheimer, 2001). This degree of response flushing is substantially less than the complete resetting of RS mechanisms discussed above and may not be sufficient to make Task 1 and Task 2 RS processes discrete. Given this, an equivalent experimental problem might now become whether Task 2 performance can be influenced by R2 information generated during Task 1 performance, in comparison with our Task 2-to-Task 1 response priming investigations. It is not clear what the former effect might look like, nor how one might differentiate such an effect experimentally.

It should be emphasized that some degree of flushing is necessary in Logan and Gordon's (2001) ECTVA model to prevent response perseveration, but the exact amount of flushing can vary over quite a wide range (e.g., from $50 \%$ to $99 \%$ ). At any point in this range, reliable information about R2 is available to influence R1, and after flushing, a substantial amount of this initial R2 information is still present to influence $\mathrm{R} 2$ selection. In contrast, there is no reason to assume flushing in the RSB model except to find a way of saving the discreteness assumption in the face of evidence that R2 information is generated (or selected) during R1 processing. Moreover, RSB theory requires a specific amount of flushing - $100 \%$ - to preserve the discreteness assumption.

One final model of PRP performance that has garnered interest of late is that proposed by Hommel (1998), which has been subsequently considered and discussed by Lien and Proctor (2002), among others. This model essentially divides the response-selection stage of the canonical RSB model (Pashler, 1984, 1994; Pashler \& Johnston, 1998) into two distinct and separate processes: an initial process of response activation, which is characterized as fast and relatively automatic and may operate for both tasks in parallel, and a later stage of final response selection, which is characterized as slow, effortful, and obligatorily serial and discrete, akin to the canonical response-selection stage in Pashler's RSB model. This new approach allows one to account for data showing Task 1 RS being influenced by R2 information, via crosstalk between simultaneously active response-activation processes, while maintaining the notion of a structural bottleneck in response selection proper.

In considering Hommel's (1998) model, it seems to us that concessions made with regard to degrees of parallelism in the computation of response information for the two tasks may lead to one or more challenges of RSB theory's specific assumptions. As discussed above, considering the issue of parallel versus discrete RS processes in informational terms may be quite useful. If R2 information generated in parallel with Task 1 RS does not contribute significantly to the computation of the Task 2 responsebecause the information is too error-full or too weak, or because it is flushed from the system after Task 1 RS finishes - then Task 2 RS essentially starts from scratch after Task $1 \mathrm{RS}$ is completed and may be considered to be discrete and serial with regard to Task 1 RS, maintaining the RS bottleneck. Hommel's two-part response process model does not make an issue of such informational possibilities. Indeed, the model appears to suggest that information about both R1 and R2 is generated in parallel in the earlier response-activation stage, and that the later serial final-response-selection stage provides further processing and gating of this information. Considering this model in informational terms as above, it seems clear that information generated during response activation of 
Task 2 is used to compute the eventual response for that task. That Task 2 response information generated during Task 1 RS influences Task 2 RS suggests that the eventual selection of responses for the two tasks is not discrete. Such informational discreteness has been suggested as a serious test for the more general family of PRP models (discussed above) and proposed as a reasonable way to preserve RSB theory in the face of R2-to-R1 compatibility data. Hommel's model would appear to directly violate the informational discreteness that has been suggested to be a necessity of RSB theory, despite claims in that article to preserve it.

This is a somewhat provocative argument, and one likely reply might be that regardless of the informational theory above, Hommel's (1998) two-part response model still embodies an obvious bottleneck in response selection (the final-response-selection process), the particular processing of which cannot begin for Task 2 until it is completed for Task 1 . We recognize this argument, most specifically the issue of particular types of processing being unable to proceed for both tasks in parallel (indeed, this is the very definition of the bottleneck). Given this argument, however, we suggest that if one concedes the parallel operation of response-activation processes that contribute directly to the eventual computation of the responses for their respective tasks, one is left with a final-response-selection stage that has substantially less to do than in Pashler and Johnston's (1998) canonical RSB model.

From here, there are several forms of the argument, some stronger than others. If one considers response selection in terms of exemplar-based random-walk or similar processes (see, e.g., Nosofsky \& Palmeri, 1997), there is little distinction between the accumulation of information and an eventual selection or decision process - response selection per se is simply the point at which accrual of information reaches threshold, at which point the selection process is complete. The information-accrual part of the random-walk process, in many ways equivalent to the response-activation phase of Hommel's (1998) two-part response model, takes a substantial amount of time to complete, whereas crossing the threshold occurs essentially instantaneously, at a single point in time. From this viewpoint, the demonstration of simultaneous generation of R2 and R1 information makes a strong case for the parallel operation of response-selection processes of both tasks in the PRP paradigm. As discussed above, Logan and Gordon's (2001) ECTVA model employs a variant of this process in successfully modeling a number of aspects of PRP task performance in a fundamentally parallel system.

To take a less extreme view, one is left with the question of what the function of final response selection actually is, if much of the work of accruing response-related information is already completed by response activation; in essence, what processing is left for there to be a bottleneck in? An obvious answer might involve the benefits of selective, controlled attention allocated to the RS processes of the task being performed, with the attendant improvement of performance such allocation typically affords. Indeed, we agree that this is likely a large component of what it means to perform Hommel's (1998) final response selection. In this guise of controlled attention, however, the latter process begins to resemble a somewhat more flexible, functional, perhaps even redirectable and not necessarily obligatory bottleneck - a significant and vital departure from Pashler and Johnston's (1998) RSB theory, which declares a structural and fundamentally obligatory bottleneck. We state these differences here in their most extreme potential terms and do not suggest that such views are implied by Hommel. However, we believe they make an important point. The final-response-selection process in Hommel's model has substantially fewer processing requirements to fulfill than does the response-selection stage in Pashler and Johnston's RSB theory, and commensurately it has far fewer potential constraints on its instantiation and behavior. As an illustration, one might conceive of two tasks with a series of processes leading in turn from perceptual analysis, to response activation, to response execution, all running in parallel. A behavioral bottleneck would be observed with a flexible, functional controlled-attention process differentially attending to and modulating the response-activation process of each task in turn. In most instances, this would be behaviorally identical to Hommel's model, although it is a significant departure from Pashler's RSB theory.

\section{The Nature of Task Sets}

The finding of parallel semantic-retrieval and responseselection processes in different-task-set trials implies that the task sets for two different tasks were simultaneously instantiated and utilized to allow such performance. In addition, substantial set-switching costs were observed in different-task-set trials, as compared with same-taskset trials, in Experiment 1 (112 $\mathrm{msec}$ and $237 \mathrm{msec}$ for Tasks 1 and 2, respectively). These findings raise a number of fundamental questions about the nature of task sets themselves. Previous research has suggested that only a single task set can be instantiated at one time (Sudevan \& Taylor, 1987) and that task sets must (by definition) be reconfigured between tasks to allow the performance of a different task (Allport, Styles, \& Hsieh, 1994; Meiran, 1996; Rogers \& Monsell, 1995). Pashler (2000) has previously suggested that two task sets may be activated simultaneously, although from different types of data than those presented here. One question here concerns the distinction between the possibility that two task sets are actively maintained in parallel and the idea that one task set is actively maintained while another persists because activation of the prior task has not yet decayed. Though the latter possibility bears some resemblance to Allport et al.'s task set inertia hypothesis, more recent research (e.g., Wylie \& Allport, 2000) suggests that so-called "residual" switch costs represent longer term effects of trial-by-trial strengthening of specific S-R mappings, and not the effects of control processes. Our data do not directly favor one conception or the other, although research pursuing such questions may be possible using methods based on those developed here. 


\section{Conclusions}

The data presented here demonstrate Task 2 response information influencing the response-selection process of Task 1 in a typical PRP paradigm, suggesting that response-selection processes may operate in parallel for both tasks of a dual-task pair. Such findings would be inconsistent with the discrete-stage processing assumption of the locus-of-slack logic, upon which RSB theory (Pashler, 1984, 1994; Pashler \& Johnston, 1998) is based. The framing of these data in the two-stage response-selection model of Hommel (1998), in which initial response activation is parallel and subsequent final response selection is serial and discrete between tasks, suggests a less constrained, potentially more flexible notion of response selection, which appears increasingly similar to the notion of a more functional central bottleneck, grounded in the mechanisms of selective attention. Whatever the fate of the particular models discussed here, our data suggest that responseselection processes for tasks in the PRP paradigm may operate in a substantially more parallel fashion than the canonical RSB theory proposes. Whether such data may pose a direct challenge to RSB theory itself will likely depend on subsequent investigations of informational discreteness in Task 2 response selection-whether Task 2 response information generated during Task 1 response selection may contribute to eventual computation of the Task 2 response.

\section{REFERENCES}

Allport, D. A., Styles, E. A., \& Hsieh, S. (1994). Shifting intentional set: Exploring the dynamic control of tasks. In C. Umiltà \& M. Moscovitch (Eds.), Attention and performance XV: Conscious and nonconscious information processing (pp. 421-452). Cambridge, MA: MIT Press.

Ashby, F. G. (1982). Deriving exact predictions from the cascade model. Psychological Review, 89, 599-607.

Bundesen, C. (1990). A theory of visual attention. Psychological Review, 97, 523-547.

CARrier, M. L., \& PAshler, H. (1995). Attentional limits in memory retrieval. Journal of Experimental Psychology: Learning, Memory, \& Cognition, 21, 1339-1348.

Coles, M. G. H., Gratton, G., Bashore, T. R., Eriksen, C. W., \& Donchin, E. (1985). A psychophysiological investigation of the continuous flow model of human information processing. Journal of Experimental Psychology: Human Perception \& Performance, 11, 529-553.

Craft, J. L., \& Simon, J. R. (1970). Processing symbolic information from a visual display: Interference from an irrelevant directional cue. Journal of Experimental Psychology, 83, 415-420.

ERIKSEN, B. A., \& ERIKSEN, C. W. (1974). Effects of noise letters upon the identification of a target letter in a nonsearch task. Perception \& Psychophysics, 16, 143-149.

Hirst, W., \& KalmaR, D. (1987). Characterizing attentional resources. Journal of Experimental Psychology: General, 116, 68-81.

Hommel, B. (1998). Automatic stimulus-response translation in dualtask performance. Journal of Experimental Psychology: Human Perception \& Performance, 24, 1368-1384.

Hommel, B., Müsseler, J., Aschersleben, G., \& Prinz, W. (2001). The theory of event coding (TEC): A framework for perception and action planning. Behavioral \& Brain Sciences, 24, 849-878.

Lien, M.-C., \& Proctor, R. W. (2000). Multiple spatial correspondence effects on dual-task performance. Journal of Experimental Psychology: Human Perception \& Performance, 26, 1260-1280.

Lien, M.-C., \& Proctor, R. W. (2002). Stimulus-response compat- ibility and psychological refractory period effects: Implications for response selection. Psychonomic Bulletin \& Review, 9, 212-238.

LogAN, G. D. (1980). Attention and automaticity in Stroop and priming tasks: Theory and data. Cognitive Psychology, 12, 523-553.

Logan, G. D., \& Delheimer, J. A. (2001). Parallel memory retrieval in dual-task situations: II. Episodic memory. Journal of Experimental Psychology: Learning, Memory, \& Cognition, 27, 668-685.

LOGAN, G. D., \& GoRdon, R. D. (2001). Executive control of visual attention in dual-task situations. Psychological Review, 108, 393-434.

Logan, G. D., \& Schulkind, M. D. (2000). Parallel memory retrieval in dual-task situations: I. Semantic memory. Journal of Experimental Psychology: Human Perception \& Performance, 26, 1072-1090.

Lu, C.-H., \& Proctor, R. W. (1995). The influence of irrelevant location information on performance: A review of the Simon and spatial Stroop effects. Psychonomic Bulletin \& Review, 2, 174-207.

MCClelland, J. L. (1979). On the time relations of mental processes: An examination of systems of processes in cascade. Psychological Review, 86, 287-330.

MeIran, N. (1996). Reconfiguration of processing mode prior to task performance. Journal of Experimental Psychology: Learning, Memory, \& Cognition, 22, 1423-1442.

Meyer, D. E., \& Kieras, D. E. (1997a). A computational theory of executive cognitive processes and multiple-task performance: 1 . Basic mechanisms. Psychological Review, 104, 3-65.

Meyer, D. E., \& Kieras, D. E. (1997b). A computational theory of executive cognitive processes and multiple-task performance: 2 . Accounts of psychological refractory-period phenomena. Psychological Review, 104, 749-791.

NAVON, D., \& MilLER, J. (1987). Role of outcome conflict in dual-task interference. Journal of Experimental Psychology: Human Perception \& Performance, 13, 438-448.

Navon, D., \& Miller, J. (2002). Queuing or sharing? A critical evaluation of the single-bottleneck notion. Cognitive Psychology, 44, 193251.

Nosofsky, R. M., \& Palmeri, T. J. (1997). An exemplar-based random walk model of speeded classification. Psychological Review, 104, 266-300.

PASHLER, H. (1984). Processing stages in overlapping tasks: Evidence for a central bottleneck. Journal of Experimental Psychology: Human Perception \& Performance, 10, 358-377.

Pashler, H. (1994). Dual-task interference in simple tasks: Data and theory. Psychological Bulletin, 116, 220-244.

PAshler, H. (2000). Task switching and multitask performance. In S. Monsell \& J. Driver (Eds.), Control of cognitive processes: Attention and performance XVIII (pp. 277-309). Cambridge, MA: MIT Press.

Pashler, H., \& Johnston, J. C. (1998). Attentional limitations in dualtask performance. In H. Pashler (Ed.), Attention (pp. 155-189). Hove, U.K.: Psychology Press.

Rogers, R. D., \& Monsell, S. (1995). Costs of a predictable switch between simple cognitive tasks. Journal of Experimental Psychology: General, 124, 207-231.

SCHWEICKERT, R. (1978). A critical path generalization of the additive factors method: Analysis of a Stroop task. Journal of Mathematical Psychology, 18, 105-139.

SCHWEICKERT, R., \& TownSEND, J. T. (1989). A trichotomy: Interactions of factors prolonging sequential and concurrent mental processes in stochastic discrete mental (PERT) networks. Journal of Mathematical Psychology, 33, 328-347.

Sternberg, S. (1969). The discovery of processing stages: Extensions of Donders' method. In W. G. Koster (Ed.), Attention and performance II (pp. 276-315). Amsterdam: North-Holland.

Stoet, G., \& Hommel, B. (1999). Action planning and the temporal binding of response codes. Journal of Experimental Psychology: Human Perception \& Performance, 25, 1625-1640.

Sudevan, P., \& TAYLOR, D. A. (1987). The cuing and priming of cognitive operations. Journal of Experimental Psychology: Human Perception \& Performance, 13, 89-103.

Telford, C. W. (1931). The refractory phase of voluntary and associative responses. Journal of Experimental Psychology, 14, 1-36.

Tombu, M., \& Joliceur, P. (2003). A central capacity sharing model of 
dual-task performance. Journal of Experimental Psychology: Human Perception \& Performance, 29, 3-18.

Townsend, J. T., \& SChweickert, R. (1989). Toward the trichotomy method of reaction times: Laying the foundation of stochastic mental networks. Journal of Mathematical Psychology, 33, 309-327.

WYlie, G., \& Allport, A. (2000). Task switching and the measurement of "switch costs." Psychological Research, 63, 212-233.

\section{NOTE}

1. We are grateful to Hal Pashler and Pierre Jolicœur for suggesting several variants of these models to us.

(Manuscript received November 10, 2003; revision accepted for publication May 2, 2005.) 